TRANSACTIONS OF THE

AMERICAN MATHEMATICAL SOCIETY

Volume 364, Number 11, November 2012, Pages 5969-5994

S 0002-9947(2012)05605-4

Article electronically published on May 30, 2012

\title{
CSR EXPANSIONS OF MATRIX POWERS IN MAX ALGEBRA
}

\author{
SERGEĬ SERGEEV AND HANS SCHNEIDER
}

\begin{abstract}
We study the behavior of max-algebraic powers of a reducible nonnegative matrix $A \in \mathbb{R}_{+}^{n \times n}$. We show that for $t \geq 3 n^{2}$, the powers $A^{t}$ can be expanded in max-algebraic sums of terms of the form $C S^{t} R$, where $C$ and $R$ are extracted from columns and rows of certain Kleene stars, and $S$ is diagonally similar to a Boolean matrix. We study the properties of individual terms and show that all terms, for a given $t \geq 3 n^{2}$, can be found in $O\left(n^{4} \log n\right)$ operations. We show that the powers have a well-defined ultimate behavior, where certain terms are totally or partially suppressed, thus leading to ultimate $C S^{t} R$ terms and the corresponding ultimate expansion. We apply this expansion to the question whether $\left\{A^{t} y, t \geq 0\right\}$ is ultimately linear periodic for each starting vector $y$, showing that this question can also be answered in $O\left(n^{4} \log n\right)$ time. We give examples illustrating our main results.
\end{abstract}

\section{INTRODUCTION}

By max algebra we understand the analogue of linear algebra developed over the max-times semiring $\mathbb{R}_{\max , \times}$ which is the set of nonnegative numbers $\mathbb{R}_{+}$equipped with the operations of "addition" $a \oplus b:=\max (a, b)$ and the ordinary multiplication $a \otimes b:=a \times b$. The zero and unity of this semiring coincide with the usual 0 and 1. The operations of the semiring are extended to the nonnegative matrices and vectors in the same way as in conventional linear algebra. That is, if $A=\left(a_{i j}\right)$, $B=\left(b_{i j}\right)$ and $C=\left(c_{i j}\right)$ are matrices of compatible sizes with entries from $\mathbb{R}_{+}$, we write $C=A \oplus B$ if $c_{i j}=a_{i j} \oplus b_{i j}$ for all $i, j$ and $C=A \otimes B$ if $c_{i j}=\bigoplus_{k} a_{i k} b_{k j}=$ $\max _{k}\left(a_{i k} b_{k j}\right)$ for all $i, j$.

If $A$ is a square matrix over $\mathbb{R}_{+}$, then the iterated product $A \otimes A \otimes \ldots \otimes A$ in which the symbol $A$ appears $k$ times will be denoted by $A^{k}$. These are the max-algebraic powers of nonnegative matrices, the main object of our study.

The max-plus semiring $\mathbb{R}_{\max ,+}=(\mathbb{R} \cup\{-\infty\}, \oplus=\max , \otimes=+)$, developed over the set of real numbers $\mathbb{R}$ with adjoined element $-\infty$ and the ordinary addition playing the role of multiplication, is another isomorphic "realization" of max algebra. In particular, $x \mapsto \exp (x)$ yields an isomorphism between $\mathbb{R}_{\max ,+}$ and $\mathbb{R}_{\max , x}$. In the max-plus setting, the zero element is $-\infty$ and the unity is 0 .

The main results of this paper are formulated in the max-times setting, since some important connections with nonnegative and Boolean matrices are more transparent there. However, the max-plus setting is left for the examples in the last section, in order to appeal to the readers who work with max-plus algebra and applications in scheduling problems and discrete event systems [1, [5, 8, 17.

Received by the editors December 29, 2009 and, in revised form, March 1, 2011.

2010 Mathematics Subject Classification. Primary 15A80, 15A23, 15A21.

This work was supported by EPSRC grant RRAH12809 and RFBR grant 08-01-00601. 
The Cyclicity Theorem is a classical result of max algebra. In the max-times setting it means that the max-algebraic powers of any irreducible nonnegative matrix are ultimately periodic (up to a scalar multiple), with the period equal to cyclicity of the critical graph. This theorem can be found in Heidergott et al. 17, Theorem 3.9]; see also Baccelli et al. [1, Theorem 3.109] and Cuninghame-Green [8, Theorem 27-6] (all stated in the max-plus setting). However, the length of the preperiodic part can be arbitrarily large, and the result does not have an evident extension to reducible matrices.

The behavior of max-algebraic powers of reducible matrices relies on connections between their strongly connected components and the hierarchy of their maxalgebraic eigenvalues. This has been well described in a monograph of Gavalec [16]; see also $9,15,20$.

The relation of the Cyclicity Theorem to the periodicity of Boolean matrices is understood but not commonly and explicitly used in max algebra. For instance the construction of cyclic classes [2, 3] appears in the proof of Lemma 3.3 in [17] (without references to the literature on Boolean matrices). This relation becomes particularly apparent after application of a certain $D^{-1} A D$ similarity scaling called visualization in 25, 26]; see also [10, 11. Semančíková 22, 23] realized that the cyclic classes of Boolean matrices are helpful in treating computational complexity of the periodicity problems in max-min algebra, with analogous max-algebraic applications in mind 24.

A result by Nachtigall 21 states that, though the length of the preperiodic part cannot be polynomially bounded, the behavior of matrix powers after $O\left(n^{2}\right)$ can be represented as max-algebraic sums of matrices from certain periodic sequences. Molnárová [19] studies this Nachtigall expansion further, showing that for a given matrix power after $O\left(n^{2}\right)$ the representing Nachtigall matrices can be computed in $O\left(n^{5}\right)$ time.

In the general reducible case, the sequences of entries $\left\{a_{i j}^{t}, t \geq 0\right\}$ of $A^{t}$ are ultimately generalized periodic 9 , 20, meaning that for $t \geq T$, where $T$ is sufficiently large, they may consist of several ultimately periodic subsequences which grow with different rates. Gavalec [15, 16] showed that deciding whether $\left\{a_{i j}^{t}, t \geq 0\right\}$ is ultimately periodic is in general NP-hard. In a related study, Butkovič et al. [5, 6] considered robust matrices, such that for any given $x$ the sequence $\left\{A^{t} x\right\}$ is ultimately periodic with period 1 . The conditions formulated in [6] can be verified in polynomial time, which suggests that such "global" periodicity questions must be tractable.

The main goal of this paper is to find a common ground for the above pieces of knowledge on matrix periodicity in max algebra. We introduce the concept of $C S R$ expansion, in which a matrix power $A^{t}$ is represented as the max-algebraic sum of terms of the form $C S^{t} R$, called $C S R$ products. Here $C$ and $R$ have been extracted from columns and rows of certain Kleene star (the same for both), and $S$ can be made Boolean by a similarity scaling $D^{-1} S D$. The matrix $C R$ appeared previously as the spectral projector [1, 7, and $S$ typically arises as the incidence matrix of a critical graph.

After giving necessary preliminaries in Section 2, we start in Section 3 by studying the $C S R$ products. We show that they form a cyclic group and describe the action of this group on the underlying Kleene star. We also emphasize the path 
sense of these operators, see Theorem 3.3 thus providing a connection to the approach of $[9,15,16,20,22,23$. In Section 4 we establish the algebraic form of the Nachtigall expansion which controls the powers $A^{t}$ after $t \sim O\left(n^{2}\right)$. See Theorem 4.2. In Section 5 we show that at large $t$ certain Nachtigall terms become totally or partially suppressed by heavier ones, leading to the ultimate expansion of matrix powers. This result, see Theorem 5.6, can be understood as a generalization of the Cyclicity Theorem to the reducible case. In Section 6 we treat the computational complexity of computing the terms of CSR expansions for a given matrix power, showing that in general this can be done in no more than $O\left(n^{4} \log n\right)$ operations. In Section 7, which extends the results of Butkovič et al. [5, 6] on robust matrices, we describe orbit periodic matrices $A$, i.e., such that the orbit $A^{t} y$ is ultimately linear periodic for all initial vectors $y$; see Theorem 7.6. We use the ultimate expansion to show that the conditions for orbit periodicity can be verified in no more than $O\left(n^{4} \log n\right)$ operations; see Theorem 7.8 and its corollary. We conclude with Section 8 which contains some examples given in the max-plus setting.

\section{Preliminaries}

In this section we recall some important notions of max algebra. These are the maximum cycle geometric mean, the critical graph and the Kleene star. We close the section with nonnegative similarity scalings and cyclic classes of the critical graph.

Let $A=\left(a_{i j}\right) \in \mathbb{R}_{+}^{n \times n}$. The weighted digraph $\mathcal{D}(A)=(N(A), E(A))$, with the set of nodes $N(A)=\{1, \ldots, n\}$ and the set of edges $E(A)=\left\{(i, j) \mid a_{i j} \neq 0\right\}$ with weights $w(i, j)=a_{i j}$, is called the digraph associated with $A$. Suppose that $P=\left(i_{1}, \ldots, i_{p}\right)$ is a path in $D_{A}$. Then the weight of $P$ is defined to be $w(P)=$ $a_{i_{1} i_{2}} a_{i_{2} i_{3}} \ldots a_{i_{p-1} i_{p}}$ if $p>1$, and 1 if $p=1$. If $i_{1}=i_{p}$, then $P$ is called a cycle. The length of $P$, denoted by $l(P)$, is the number of edges in $P$ (it equals $p-1$ here).

When any two nodes in $\mathcal{D}(A)$ can be connected to each other by paths, the matrix $A$ is called irreducible. Otherwise, it is called reducible. In the reducible case, there are some (maximal) strongly connected components of $\mathcal{D}(A)$, and a number of nodes that do not belong to any cycle. We will refer to such nodes as trivial components of $\mathcal{D}(A)$.

The maximum cycle geometric mean of $A$, further denoted by $\lambda(A)$, is defined by the formula

$$
\lambda(A)=\max _{P_{c}}\left(w\left(P_{c}\right)\right)^{1 / k},
$$

where the maximization is taken over all cycles $P_{c}=\left(i_{1}, \ldots, i_{k}\right)$, for $k=1, \ldots, n$, in the digraph $\mathcal{D}(A)$.

The Cyclicity Theorem ([17, Theorem 3.9]; see also [1, 5, 8]) states that if $A$ is irreducible, then after a certain time $T(A)$, there exists a number $\gamma$ such that $A^{t+\gamma}=\lambda^{\gamma}(A) A^{t}$ for all $t \geq T(A)$. Thus $\lambda$ is the ultimate growth rate of matrix powers in this case. If $\lambda=1$, then $A^{t+\gamma}=A^{t}$ for $t \geq T(A)$, in which case we say that $\left\{A^{t}, t \geq 0\right\}$ is ultimately periodic.

Remarkably $\lambda(A)$ is also the largest max-algebraic eigenvalue of $A$, meaning the largest number $\lambda$ for which there exists a nonzero $x \in \mathbb{R}_{+}^{n}$ such that $A \otimes x=\lambda x$; see [1, 4, 5, 8, 17] and the references therein.

The operation of taking the maximal cycle geometric mean (m.c.g.m. for short) is homogeneous: $\lambda(\alpha A)=\alpha \lambda(A)$. Hence any matrix, which has $\lambda(A) \neq 0$ meaning 
that $\mathcal{D}(A)$ is not acyclic, can be scaled so that $\lambda(A / \lambda(A))=1$. Following [4, matrix $A \in \mathbb{R}_{+}^{n \times n}$ with $\lambda(A)=1$ will be called definite.

A cycle $P_{c}=\left(i_{1}, \ldots, i_{k}\right)$ in $\mathcal{D}(A)$ is called critical if $\left(w\left(P_{c}\right)\right)^{1 / k}=\lambda(A)$. Every node and edge that belongs to a critical cycle is called critical. The set of critical nodes is denoted by $N_{c}(A)$; the set of critical edges is denoted by $E_{c}(A)$. The critical digraph of $A$, further denoted by $\mathcal{C}(A)=\left(N_{c}(A), E_{c}(A)\right)$, is the digraph which consists of all critical nodes and critical edges of $\mathcal{D}(A)$.

The cyclicity of an irreducible graph is defined as the g.c.d. (greatest common divisor) of the lengths of all its simple cycles. The critical graph defined above may have several strongly connected components, and in this case the cyclicity is the l.c.m. (least common multiple) of their cyclicities. This gives the number $\gamma$ which appears in the Cyclicity Theorem [1, 17, and it can be shown that the ultimate period cannot be less than $\gamma$ (in particular, this follows from the approach of the present paper).

There is no obvious subtraction in max algebra; however, we have an analogue of $(I-A)^{-1}$ defined by

$$
A^{*}:=I \oplus A \oplus A^{2} \oplus \ldots,
$$

where $I$ is the identity matrix. This series converges to a finite matrix if and only if $\lambda(A) \leq 1$ [1, 4, 8, 17, and then this matrix $A^{*}=\left(a_{i j}^{*}\right)$ is called the Kleene star of $A$. This matrix has properties $\left(A^{*}\right)^{2}=A^{*}$ and, clearly, $A^{*} \geq I$. It is important that the entries of max-algebraic powers $A^{k}=\left(a_{i j}^{k}\right)$ express the maximal weights of certain paths: $a_{i j}^{k}$ is equal to the greatest weight of paths $P$ that connect $i$ to $j$ and have length $k$. The entry $a_{i j}^{*}$ for $i \neq j$ is equal to the greatest weight of paths that connect $i$ to $j$ with no restriction on their lengths.

As in the nonnegative linear algebra, we have only few invertible matrices, in the sense of the existence of (nonnegative) $A^{-1}$ such that $A^{-1} \otimes A=A \otimes A^{-1}=I$. More precisely, such matrices can be diagonal matrices

$$
X=\operatorname{diag}(x):=\left(\begin{array}{ccc}
x_{1} & \ldots & 0 \\
\vdots & \ddots & \vdots \\
0 & \ldots & x_{n}
\end{array}\right)
$$

for a positive $x=\left(x_{1}, \ldots, x_{n}\right)$, or monomial matrices obtained from the diagonal matrices by permutations of rows or columns. Nevertheless, such matrices give rise to very convenient diagonal similarity scalings $A \mapsto X^{-1} A X$. Such transformations do not change $\lambda(A)$ and $\mathcal{C}(A)$ [12. They commute with max-algebraic multiplication of matrices and hence with the operation of taking the Kleene star. Geometrically, they correspond to automorphisms of $\mathbb{R}_{+}^{n}$, both in the case of max algebra and in the case of nonnegative linear algebra. The importance of such scalings in max algebra has already been emphasized in 8 , Ch. 28.

By an observation of Fiedler and Pták [13, for any definite matrix $A$ there is a scaling $X$ such that $X^{-1} A X$ is visualized, meaning that all critical entries of the matrix equal 1 and all the rest are less than or equal to 1 . It is also possible to make $X^{-1} A X$ strictly visualized [26, meaning that only critical entries are equal to 1 . If a matrix is visualized, or strictly visualized, the same is true for all powers of this matrix, meaning that the critical graph can be seen as a Boolean matrix that "lives by itself". Thus there is a clear connection to the powers of Boolean matrices. 
The periodicity of powers of Boolean matrices is ruled by cyclic classes [2], also known as imprimitivity sets [3, which we explain below. We note that this notion has already appeared in a work of Frobenius [14].

Proposition 2.1 (e.g. Brualdi-Ryser [3]). Let $G=(N, E)$ be a strongly connected digraph with cyclicity $\gamma_{G}$. Then the lengths of any two paths connecting $i \in N$ to $j \in N$ (with $i, j$ fixed) are congruent modulo $\gamma_{G}$.

Proposition 2.1 implies that the following equivalence relation can be defined: $i \sim$ $j$ if there exists a path $P$ from $i$ to $j$ such that $l(P) \equiv 0\left(\bmod \gamma_{G}\right)$. The equivalence classes of $G$ with respect to this relation are called cyclic classes $[2,22,[23$. The cyclic class of $i$ will be denoted by $[i]$.

Consider the following access relations between cyclic classes: $[i] \rightarrow_{t}[j]$ if there exists a path $P$ from a node in $[i]$ to a node in $[j]$ such that $l(P) \equiv t\left(\bmod \gamma_{G}\right)$. In this case, a path $P$ with $l(P) \equiv t\left(\bmod \gamma_{G}\right)$ exists between any node in $[i]$ and any node in $[j]$. Further, by Proposition 2.1 , the length of any path between a node in $[i]$ and a node in $[j]$ is congruent to $t$, so the relation $[i] \rightarrow_{t}[j]$ is well defined.

Cyclic classes can be computed in $O(|E|)$ time by Balcer-Veinott digraph condensation [2], where $|E|$ denotes the number of edges in $G$. At each step of this algorithm, we look for all edges which issue from a certain node $i$, and condense all end nodes of these edges into a single node. Another efficient algorithm is described in 3 .

Let $S=\left(s_{i j}\right)$ be the incidence matrix of $G$, meaning that $s_{i j}=1$ if $(i, j) \in E$ and $s_{i j}=0$ otherwise.

The ultimate periodicity of such Boolean matrices has been well studied. If $\gamma_{G}=1$, then the periodicity of $S^{t}$ starts latest after the Wielandt number $W(n):=$ $(n-1)^{2}+1$ [3, 18]. This bound is sharp and is due to Wielandt [28].

If $\gamma>1$, then there are even better sharp bounds due to Schwartz [27. Assume w.l.o.g. that $S$ is irreducible, and let $n=\alpha \gamma+t$. If $\alpha>1$, then the periodicity starts at most after $W(\alpha) \gamma+t$ which does not exceed $\frac{n^{2}}{\gamma}+\gamma$. If $\alpha=1$, then it starts almost "straightaway", after at most $\max (1, t)$.

\section{CSR PRODUCTS}

In this section, given a nonnegative matrix $A \in \mathbb{R}_{+}^{n \times n}$, we consider max-algebraic products of the form $C S^{t} R$, where $S$ is associated with some subdigraph of the critical graph $\mathcal{C}(A)$, and matrices $C$ and $R$ are extracted from a certain Kleene star related to $A$. We show that these products form a cyclic group and study their periodic properties. We also show that $C S^{t} R$ are related to a distinguished set of paths, which we call $\mathcal{C}$-heavy.

We start with a remark that the concept of cyclic classes discussed in Section 2 can be generalized to completely reducible digraphs, which consist of (possibly several) strongly connected components, not connected with each other. Importantly, the critical digraph of any $A \in \mathbb{R}_{+}^{n \times n}$ with $\lambda(A)>0$ is completely reducible.

Let $A \in \mathbb{R}_{+}^{n \times n}$ have $\lambda(A)=1$. Consider any completely reducible subdigraph $\mathcal{C}=\left(N_{c}, E_{c}\right)$ of $\mathcal{C}(A)$. In particular, $\mathcal{C}$ may consist of several disjoint cycles of $\mathcal{C}(A)$, or we can take a component of $\mathcal{C}(A)$, or we may just have $\mathcal{C}=\mathcal{C}(A)$. Denote by $\gamma$ the cyclicity of $\mathcal{C}$ and take $B:=\left(A^{\gamma}\right)^{*}$. Define the matrices $C=\left(c_{i j}\right) \in \mathbb{R}_{+}^{n \times n}$, 


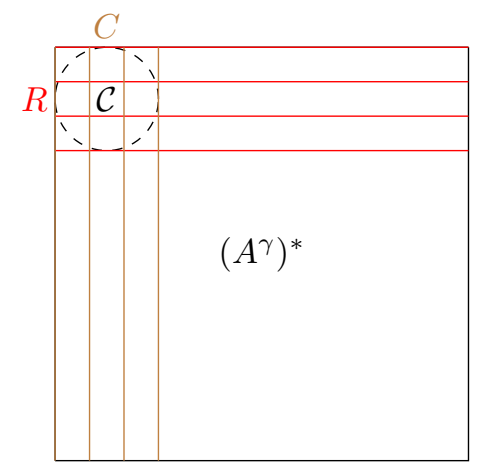

Figure 1. The scheme of $C$ and $R$ defined in (3.1)

$R=\left(r_{i j}\right) \in \mathbb{R}_{+}^{n \times n}$ and $S=\left(s_{i j}\right) \in \mathbb{R}_{+}^{n \times n}$ by

$$
\begin{aligned}
c_{i j} & =\left\{\begin{array}{ll}
b_{i j}, & \text { if } j \in N_{c}, \\
0, & \text { otherwise, }
\end{array} \quad r_{i j}= \begin{cases}b_{i j}, & \text { if } i \in N_{c}, \\
0, & \text { otherwise, }\end{cases} \right. \\
s_{i j} & = \begin{cases}a_{i j}, & \text { if }(i, j) \in E_{c}, \\
0, & \text { otherwise. }\end{cases}
\end{aligned}
$$

The nonzero entries of $C$, respectively $R$, can only be in the submatrix of $B=\left(A^{\gamma}\right)^{*}$ extracted from columns, respectively rows, in $N_{c}$. All nonzero entries of $S$ are in the principal submatrix $S_{N_{c} N_{c}}$ extracted from rows and columns in $N_{c}$. See Figure 1 for a schematic display.

We show in the next proposition that $S$ can be assumed to be $0-1$. It can also be deduced from the results in [12].

Proposition 3.1. Let $A \in \mathbb{R}_{+}^{n \times n}$ have $\lambda(A)=1$ and let $S$ be defined as above. There exists a positive $z \in \mathbb{R}_{+}^{n}$ such that $D^{-1} S D$ for $D:=\operatorname{diag}(z)$ is a $0-1$ matrix.

Proof. As $\lambda(S)=1$, we can take $z:=\bigoplus_{j=1}^{n} S_{\cdot j}^{*}$. This vector is positive, and observe that $S z \leq z$ since $S S^{*} \leq S^{*}$. From this and $\mathcal{D}(S)=\mathcal{C}(S)=\mathcal{C}$, it can be deduced by multiplying $z_{i}^{-1} s_{i j} z_{j} \leq 1$ along cycles that $z_{i}^{-1} s_{i j} z_{j}=1$ for all $(i, j) \in \mathcal{D}(S)$, while $z_{i}^{-1} s_{i j} z_{j}=0$ for all $(i, j) \notin \mathcal{D}(S)$.

As $S$ can be scaled to a $0-1$ matrix, we conclude that $\left\{S^{t}, t \geq 0\right\}$ becomes periodic at most after the Wielandt number $W\left(n_{c}\right)=\left(n_{c}-1\right)^{2}+1$, where $n_{c}$ is the number of nodes in $N_{c}$. A matrix $A$ will be called $S$-visualized if $S$ defined in (3.1) is Boolean. In the $S$-visualized case, the asymptotic form of $S^{t}$ for $t \geq T$ is determined by the cyclic classes of $\mathcal{C}[3]$ :

$$
s_{i j}^{t}= \begin{cases}1, & \text { if }[i] \rightarrow_{t}[j], \\ 0, & \text { otherwise. }\end{cases}
$$

Now we study the CSR products

$$
\mathcal{P}^{(t)}:=C S^{t} R,
$$

assuming that $A \in \mathbb{R}_{+}^{n \times n}$ has $\lambda(A)=1$. 
We start by the observation that if $T$ is the number after which $\left\{S^{t}, t \geq 0\right\}$ becomes periodic, then

$$
S^{l \gamma} R=R, \quad C S^{l \gamma}=C, \quad \forall l \gamma \geq T .
$$

Indeed, (3.2) implies that all diagonal entries of $S^{l \gamma}$ with indices in $N_{c}$ are 1 if $l \gamma \geq T$, which implies $S^{l \gamma} R \geq R$ and $C S^{l \gamma} \geq C$. On the other hand, $S \leq A$ and so $S^{l \gamma} \leq\left(A^{\gamma}\right)^{*}$. Further, $\left(A^{\gamma}\right)^{*} R \leq R$ and $C\left(A^{\gamma}\right)^{*} \leq C$ since $\left(A^{\gamma}\right)^{*}\left(A^{\gamma}\right)^{*}=\left(A^{\gamma}\right)^{*}$, and so $S^{l \gamma} R \leq\left(A^{\gamma}\right)^{*} R \leq R$ and $C S^{l \gamma} \leq C\left(A^{\gamma}\right)^{*} \leq C$.

As $\left\{S^{t}, t \geq T\right\}$ is periodic, so is $\left\{\mathcal{P}^{(t)}, t \geq T\right\}$. Moreover, we conclude from (3.4) that this periodicity starts from the very beginning.

Proposition 3.2 (Periodicity). $\mathcal{P}^{(t+\gamma)}=\mathcal{P}^{(t)}$ for all $t \geq 0$.

Proof. It follows from (3.4) that $\mathcal{P}^{(t+l \gamma)}=\mathcal{P}^{(t)}$ and $\mathcal{P}^{(t+(l+1) \gamma)}=\mathcal{P}^{(t+\gamma)}$ for $l \gamma \geq T$. But $\mathcal{P}^{(t+l \gamma)}=\mathcal{P}^{(t+(l+1) \gamma)}$, which implies that also $\mathcal{P}^{(t+\gamma)}=\mathcal{P}^{(t)}$.

It is also useful to understand the meaning of $\mathcal{P}^{(t)}$ in terms of paths. Given a set of paths $\Pi$, we denote by $w(\Pi)$ the greatest weight of paths in $\Pi$, assuming $w(\Pi)=0$ if $\Pi$ is empty. A path will be called $\mathcal{C}$-heavy if it goes through a node in $N_{c}$. The set of all $\mathcal{C}$-heavy paths on $\mathcal{D}(A)$ that connect $i$ to $j$ and have length $t$ will be denoted by $\Pi_{i j, t}^{h}$. We also denote by $\tau$ the maximal cyclicity of the components of $\mathcal{C}(A)$.

Theorem 3.3 (C-Heavy paths). Let $A \in \mathbb{R}_{+}^{n \times n}$ have $\lambda(A)=1$ and let $T \geq 0$ be such that $\left\{S^{t}, t \geq T\right\}$ is periodic.

1. For $t \geq 0$,

2. For $t \geq T+2 \tau(n-1)$,

$$
w\left(\Pi_{i j, t}^{h}\right) \leq \mathcal{P}_{i j}^{(t)} .
$$

Proof. By Proposition 3.1 there exists a diagonal matrix $D$ such that $D^{-1} A D$ is $S$-visualized. As both sides of (3.5) and (3.6) are stable under similarity scaling of $A$, we will assume that $A$ is already $S$-visualized.

1. Let $P \in \Pi_{i j, t}^{h}$ and $w(P) \neq 0$. We need to show that

$$
w(P) \leq \mathcal{P}_{i j}^{(t)}
$$

Path $P$ can be decomposed as $P=P_{\text {beg }} \circ P_{\text {end }}$, where $P_{\text {beg }}$ connects $i$ to a node $m \in N_{c}$ and $P_{\text {end }}$ connects $m$ to $j$. Adjoining to $P$ any sufficiently large number of cycles of $\mathcal{C}$ that go through $m$ and whose total length is a multiple of $\gamma$, we obtain a path $P^{\prime}$ that we can decompose as $P^{\prime}=P_{\text {beg }}^{\prime} \circ P_{\text {int }}^{\prime} \circ P_{\text {end }}^{\prime}$, where $P_{\text {beg }}^{\prime}$ connects $i$ to $m_{1} \in \mathcal{C}$ and $l\left(P_{\text {beg }}^{\prime}\right)$ is a multiple of $\gamma, P_{\text {int }}^{\prime}$ connects $m_{1}$ to $m_{2} \in N_{c}$, has length $t$ and belongs entirely to $\mathcal{C}$, and $P_{\text {end }}^{\prime}$ connects $m_{2}$ to $j$ and $l\left(P_{\text {end }}^{\prime}\right)$ is a multiple of $\gamma$. We conclude that $w\left(P_{\text {beg }}^{\prime}\right) \leq c_{i m_{1}}, w\left(P_{\text {end }}^{\prime}\right) \leq r_{m_{2} j}$ and $w\left(P_{\text {int }}^{\prime}\right)=s_{m_{1} m_{2}}^{t}=1$. We obtain $w(P)=w\left(P^{\prime}\right) \leq c_{i m_{1}} s_{m_{1} m_{2}}^{t} r_{m_{2} j}$, which implies (3.7), and hence (3.5).

2. There exist indices $m_{1}$ and $m_{2}$ such that $\mathcal{P}_{i j}^{(t)}=c_{i m_{1}} s_{m_{1} m_{2}}^{t} r_{m_{2} j}$. This is the weight of a path $P$ decomposed as $P=P_{\text {beg }} \circ P_{\text {int }} \circ P_{\text {end }}$, where $P_{\text {beg }}$ connects $i$ to $m_{1}, P_{\text {int }}$ connects $m_{1}$ to $m_{2}$ and $P_{\text {end }}$ connects $m_{2}$ to $j$. Here $P_{\text {int }}$ has length $t$ and belongs to the component of $\mathcal{C}$, which we denote by $\mathcal{T}$ and whose cyclicity we denote by $\pi$. The lengths of $P_{\text {beg }}$ and $P_{\text {end }}$ are, respectively, $l\left(P_{\text {beg }}\right)=l_{1} \pi$ 
and $l\left(P_{\text {end }}\right)=l_{2} \pi$ for some $l_{1}, l_{2}$, since $l\left(P_{\text {beg }}\right)$ and $l\left(P_{\text {end }}\right)$ are multiples of $\gamma$, and $\gamma$ is itself a multiple of $\pi$. Paths $P_{\text {beg }}$ and $P_{\text {end }}$ correspond to certain paths on $\mathcal{D}\left(A^{\pi}\right)$ with lengths $l_{1}$ and $l_{2}$. If $l_{1} \geq n$ or $l_{2} \geq n$, then we can perform cycle deletion (w.r.t. $\mathcal{D}\left(A^{\pi}\right)$ ) and obtain paths $P_{\text {beg }}^{2}$ and $P_{\text {end }}^{2}$ with lengths $k_{1} \pi$ and $k_{2} \pi$ where $k_{1}<n$ and $k_{2}<n$. For the resulting path $P^{2}:=P_{\text {beg }}^{2} \circ P_{\text {int }} \circ P_{\text {end }}^{2}$ we will have $w\left(P^{2}\right) \geq w(P)$ since $\lambda=1$. Now we have $l\left(P_{\mathrm{beg}}^{2}\right)+l\left(P_{\text {end }}^{2}\right) \leq 2 \tau(n-1)$. If $t \geq T+2 \tau(n-1)$, then the principal submatrix of $S^{t}$ corresponding to the component $\mathcal{T}$ coincides with that of $S^{t-\left(k_{1}+k_{2}\right) \pi}$, which implies that $P_{\text {int }}$ can be replaced by a path $P_{\text {int }}^{3}$ with length $t-\left(k_{1}+k_{2}\right) \pi$, so that $w\left(P^{3}\right)=w\left(P^{2}\right) \geq \mathcal{P}_{i j}^{(t)}$ where $P^{3}:=P_{\text {beg }}^{2} \circ P_{\text {int }}^{3} \circ P_{\text {end }}^{2} \in \Pi_{i j, t}^{h}$.

This "path sense" of $\mathcal{P}^{(t)}$ simplifies the proof of the following important law.

Theorem 3.4 (Group law). $\mathcal{P}^{\left(t_{1}+t_{2}\right)}=\mathcal{P}^{\left(t_{1}\right)} \mathcal{P}^{\left(t_{2}\right)}$ for all $t_{1}, t_{2} \geq 0$.

Proof. As $\left(A^{\gamma}\right)^{*}\left(A^{\gamma}\right)^{*}=\left(A^{\gamma}\right)^{*}$, we have $(R C)_{i i}=\left(A^{\gamma}\right)_{i i}^{*}=1$ for all $i \in N_{c}$, and hence $R C S \geq S$. We use this to obtain that $\mathcal{P}^{\left(t_{1}\right)} \mathcal{P}^{\left(t_{2}\right)} \geq \mathcal{P}^{\left(t_{1}+t_{2}\right)}$ :

$$
\mathcal{P}^{\left(t_{1}\right)} \mathcal{P}^{\left(t_{2}\right)}=C S^{t_{1}} R C S^{t_{2}} R \geq C S^{t_{1}+t_{2}} R=\mathcal{P}^{\left(t_{1}+t_{2}\right)} .
$$

But Theorem 3.3 implies that $\mathcal{P}^{\left(t_{1}\right)} \mathcal{P}^{\left(t_{2}\right)} \leq \mathcal{P}^{\left(t_{1}+t_{2}\right)}$ for all large enough $t_{1}, t_{2}$, since the concatenation of two $\mathcal{C}$-heavy paths is again a $\mathcal{C}$-heavy path. As $\mathcal{P}^{(t)}$ are periodic, it follows that $\mathcal{P}^{\left(t_{1}\right)} \mathcal{P}^{\left(t_{2}\right)} \leq \mathcal{P}^{\left(t_{1}+t_{2}\right)}$ for all $t_{1}, t_{2}$, and we obtain the claim combining this with the reverse inequality.

Formulas (3.2) and (3.4) imply that if $A$ is $S$-visualized, then all rows of $R$ or columns of $C$ with indices in the same cyclic class of $\mathcal{C}$ coincide. Hence, when working with $\mathcal{P}^{(t)}$ we can assume without loss of generality that all cyclic classes have just 1 element and, consequently, that $S_{N_{c} N_{c}}$ is a permutation matrix. This captures the structure of $\mathcal{P}^{(t)}$, which form a cyclic group of order $\gamma$.

As usual $e_{i}$ denotes the vector which has all coordinates equal to 0 except for the $i$ th which equals 1 . For the rows $\mathcal{P}_{i}^{(t)}$ and columns $\mathcal{P}_{. j}^{(t)}$ of $\mathcal{P}^{(t)}$ we have

$$
e_{i}^{T} \mathcal{P}^{(t)}=\mathcal{P}_{i \cdot}^{(t)}, \mathcal{P}^{(t)} e_{j}=\mathcal{P}_{\cdot j}^{(t)} .
$$

Next we study the periodicity of $\mathcal{P}^{(t)}$ in more detail. It turns out that the columns and rows of $\mathcal{P}^{(t+1)}$ with indices in $N_{c}$ can be obtained from those of $\mathcal{P}^{(t)}$ by means of a permutation on cyclic classes, while the rest of the columns (or rows) are max-linear combinations of the critical ones. We start with the following observation on the spectral projector $\mathcal{P}^{(0)}:=C R$, which can be found in [1, 7].

Lemma 3.5. $\mathcal{P}_{i .}^{(0)}=R_{i}$. and $\mathcal{P}_{\cdot i}^{(0)}=C_{. i}$ for all $i \in N_{c}$.

Proof. As $\left(A^{\gamma}\right)_{i i}^{*}=1$ for all $i \in N_{c}$, we obtain $e_{i}^{T} C=C_{i}$. $\geq e_{i}^{T}$ and $R e_{i}=R_{\text {. } i} \geq e_{i}$. We see that

$$
\begin{aligned}
& \mathcal{P}_{i \cdot}^{(0)}=e_{i}^{T} C R \geq e_{i}^{T} R=R_{i \cdot}, \\
& \mathcal{P}_{\cdot i}^{(0)}=C R e_{i} \geq C e_{i}=C_{\cdot i} .
\end{aligned}
$$

But $C R \leq\left(\left(A^{\gamma}\right)^{*}\right)^{2}=\left(A^{\gamma}\right)^{*}$, which implies the reverse inequalities $\mathcal{P}_{i}^{(0)} \leq R_{i}$. and $\mathcal{P}_{\cdot i}^{(0)} \leq C_{\cdot i}$. 
Theorem 3.6. Let $A \in \mathbb{R}_{+}^{n \times n}$ have $\lambda(A)=1$ and be $S$-visualized. If $[i] \rightarrow_{t}[j]$, then

$$
\mathcal{P}_{i \cdot}^{(t+s)}=\mathcal{P}_{j \cdot}^{(s)}, \mathcal{P}_{\cdot i}^{(s)}=\mathcal{P}_{\cdot j}^{(t+s)}
$$

for all $s, t \geq 0$.

Proof. We prove the first equality of (3.11). Using the group law we assume that $s=0$. We also assume that $S_{N_{c} N_{c}}$ is a permutation matrix; then $e_{i}^{T} S^{t}=e_{j}$. Using this and Lemma 3.5 we obtain

$$
\begin{aligned}
\mathcal{P}_{j .}^{(0)}=e_{j}^{T} \mathcal{P}^{(0)} & =e_{j}^{T} R=e_{i}^{T} S^{t} R \\
& \leq e_{i}^{T} C S^{t} R=e_{i}^{T} \mathcal{P}^{(t)}=\mathcal{P}_{i .}^{t}
\end{aligned}
$$

Analogously we have $\mathcal{P}_{i}^{(0)} \leq \mathcal{P}_{j}^{(\gamma-t)}$. Multiplying this inequality by $\mathcal{P}^{(t)}$ and using the group law and periodicity, we obtain that $\mathcal{P}_{i}^{(t)} \leq \mathcal{P}_{j}^{(\gamma)}=\mathcal{P}_{j}^{(0)}$. Combining this with (3.12) we obtain the desired property.

Corollary 3.7. Let $A \in \mathbb{R}_{+}^{n \times n}$ have $\lambda(A)=1$. Then $\mathcal{P}_{i \cdot}^{(t)}=\left(S^{t} R\right)_{i}$. and $\mathcal{P}_{\cdot i}^{(t)}=$ $\left(C S^{t}\right)_{\cdot i}$ for all $i \in N_{c}$.

Proof. We assume that $A$ is $S$-visualized, and we also assume that $S_{N_{c} N_{c}}$ is a permutation matrix. For $[i] \rightarrow_{t}[j]$, Theorem 3.6 and Lemma 3.5] imply that

$$
\begin{aligned}
& \mathcal{P}_{i \cdot}^{(t)}=e_{i}^{T} \mathcal{P}^{(t)}=e_{j}^{T} \mathcal{P}^{(0)}=e_{j}^{T} R=e_{i}^{T} S^{t} R=\left(S^{t} R\right)_{i \cdot}, \\
& \mathcal{P}_{\cdot j}^{(t)}=\mathcal{P}^{(t)} e_{j}=\mathcal{P}^{(0)} e_{i}=C e_{i}=C S^{t} e_{j}=\left(C S^{t}\right)_{\cdot j} .
\end{aligned}
$$

The claim is proved.

Corollary 3.8. Let $A \in \mathbb{R}_{+}^{n \times n}$ have $\lambda(A)=1$. For each $k=1, \ldots, n$ there exist $\alpha_{i k}$ and $\beta_{k i}$, where $k \in N_{c}$, such that

$$
\mathcal{P}_{\cdot k}^{(t)}=\bigoplus_{i \in N_{c}} \alpha_{i k} \mathcal{P}_{\cdot i}^{(t)}, \quad \mathcal{P}_{k}^{(t)}=\bigoplus_{i \in N_{c}} \beta_{k i} \mathcal{P}_{i .}^{(t)}
$$

Proof. By Corollary 3.7 we have $\mathcal{P}_{\cdot i}^{(t)}=\left(C S^{t}\right)_{{ }_{i}}$ and $\mathcal{P}_{i}^{(t)}=\left(S^{t} R\right)_{i}$. for all $i \in N_{c}$. Equation (3.13) follows directly from $\mathcal{P}^{(t)}=C S^{t} R$, the coefficients $\alpha_{i k}$ (resp. $\beta_{k i}$ ) being taken from the $k$ th column of $R$ (resp. the $k$ th row of $C$ ).

\section{NACHTIGALL EXPANSIONS}

In this section we show that the powers $A^{t}$ of $A \in \mathbb{R}_{+}^{n \times n}$ can be expanded for $t \geq 3 n^{2}$ as a sum of $C S R$ products. This establishes a more general algebraic form of the Nachtigall expansion studied in [19, 21].

Let $A=\left(a_{i j}\right) \in \mathbb{R}_{+}^{n \times n}$. Define $\lambda_{1}=\lambda(A)$, and let $\mathcal{C}_{1}=\left(N_{1}, E_{1}\right)$ be a completely reducible subdigraph of $\mathcal{C}(A)$. Set $A_{1}:=A$ and $K_{1}:=N$, where $N=\{1, \ldots, n\}$.

The elements of a Nachtigall expansion will now be defined inductively for $\mu \geq 2$. Namely, we define $K_{\mu}:=N \backslash \bigcup_{i=1}^{\mu-1} N_{i}$ and $A_{\mu}=\left(a_{i j}^{\mu}\right) \in \mathbb{R}_{+}^{n \times n}$ by

$$
a_{i j}^{\mu}= \begin{cases}a_{i j}, & \text { if } i, j \in K_{\mu} \\ 0, & \text { otherwise }\end{cases}
$$



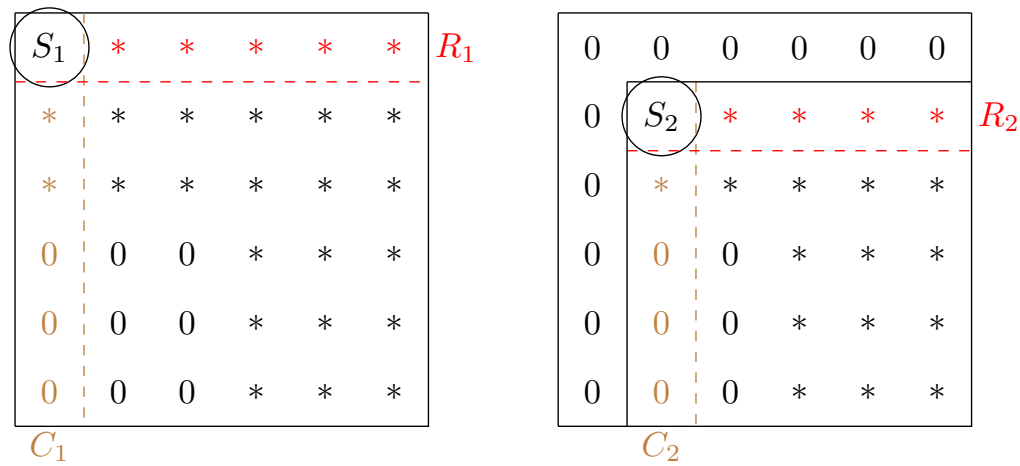

FiguRE 2. Formation of first two terms in a Nachtigall expansion: a schematic example. Note that the structure of strongly connected components of $\mathcal{D}(A)$ is of no use here.

Further define $\lambda_{\mu}:=\lambda\left(A_{\mu}\right)$. If $\lambda_{\mu}=0$, then stop; otherwise, select a completely reducible subdigraph $\mathcal{C}_{\mu}=\left(N_{\mu}, E_{\mu}\right)$ of the critical digraph $\mathcal{C}\left(A_{\mu}\right)$, and proceed as above with $\mu:=\mu+1$.

By the above procedure we define $K_{\mu}, A_{\mu}, \lambda_{\mu}$ and $\mathcal{C}_{\mu}=\left(N_{\mu}, E_{\mu}\right)$ for $\mu=$ $1, \ldots, m$, where $m \leq n$ is the last number $\mu$ such that $\lambda_{\mu}>0$.

Denote $L:=\bigcup_{i=1}^{m} N_{\mu}$ and $\bar{L}=N \backslash L$.

For each $\mu=1, \ldots, m$, let $\gamma_{\mu}$ be the cyclicity of $\mathcal{C}_{\mu}$. Since $\lambda\left(\left(A_{\mu} / \lambda_{\mu}\right)^{\gamma_{\mu}}\right)=$ $\lambda\left(A_{\mu} / \lambda_{\mu}\right)=1$, the Kleene star $B_{\mu}:=\left(\left(A_{\mu} / \lambda_{\mu}\right)^{\gamma_{\mu}}\right)^{*}$ is finite. Define the matrices $C_{\mu}=\left(c_{i j}^{\mu}\right) \in \mathbb{R}_{+}^{n \times n}, R_{\mu}=\left(r_{i j}^{\mu}\right) \in \mathbb{R}_{+}^{n \times n}$ and $S_{\mu}=\left(s_{i j}^{\mu}\right) \in \mathbb{R}_{+}^{n \times n}$ by

$$
\begin{aligned}
& c_{i j}^{\mu}=\left\{\begin{array}{ll}
b_{i j}^{\mu}, & \text { if } j \in N_{\mu}, \\
0, & \text { otherwise, }
\end{array} \quad r_{i j}^{\mu}= \begin{cases}b_{i j}^{\mu}, & \text { if } i \in N_{\mu}, \\
0, & \text { otherwise, }\end{cases} \right. \\
& s_{i j}^{\mu}= \begin{cases}a_{i j}^{\mu} / \lambda_{\mu}, & \text { if }(i, j) \in E_{\mu}, \\
0, & \text { otherwise. }\end{cases}
\end{aligned}
$$

A schematic example of a Nachtigall expansion is given in Figure 2 .

It follows from Proposition 3.1 that each $S_{\mu}$ can be scaled to a $0-1$ matrix using a certain vector denoted here by $z^{\mu}$. Note that the sets $N_{\mu}$ are pairwise disjoint. Defining $z \in \mathbb{R}_{+}^{n}$ by

$$
z_{i}= \begin{cases}z_{i}^{\mu}, & \text { if } i \in N_{\mu} \\ 1, & \text { if } i \in \bar{L},\end{cases}
$$

and letting $D:=\operatorname{diag}(z)$, we obtain that the matrix $\tilde{A}:=D^{-1} A D$ is totally $S$ visualized, meaning that all corresponding matrices $\tilde{S}_{\mu}$ are Boolean.

As $S_{\mu}$ can be scaled to be Boolean, the sequences of their max-algebraic powers $\left\{S_{\mu}^{t} \mid t \geq 0\right\}$, being powers of Boolean matrices when scaled, are ultimately periodic with periods $\gamma_{\mu}$. This periodicity starts at most after the corresponding Wielandt numbers $\left(k_{\mu}-1\right)^{2}+1$, where $k_{\mu}$ is the number of elements in $N_{\mu}$.

We proceed with some notation. Denote $\mu(i)=\mu$ if $i \in N_{\mu}$, and $\mu(i)=+\infty$ if $i \in \bar{L}$. Denote by $\Pi_{i j, t}$ the set of paths on $\mathcal{D}(A)$ which connect $i$ to $j$ and have length $t$. Denote by $\Pi_{i j, t}^{\mu}$ the set of paths $P \in \Pi_{i j, t}$ such that $\min _{i \in N_{P}} \mu(i)=\mu$, 
where $N_{P}$ is the set of nodes visited by $P$. (Note that greater values of $\mu$ correspond to smaller $\lambda_{\mu}$.) The paths in $\Pi_{i j, t}^{\mu}$ will be called $\mu$-heavy, since they are $\mathcal{C}_{\mu}$-heavy (see Section 3) in $A_{\mu}$.

Any path $P \in \Pi_{i j, t}$ with $t \geq n$ has at least one cycle. Hence there are no paths with length $t \geq n$ that visit only the nodes in $\bar{L}$, for otherwise the subdigraph of $\mathcal{D}(A)$ induced by $\bar{L}$ would contain a cycle and the number of components would be more than $m$. We can express the entries of $A^{t}=\left(a_{i j}^{t}\right)$ and $A_{\mu}^{t}=\left(a_{i j}^{\mu, t}\right)$ for $t \geq n$ as follows:

$$
\begin{aligned}
a_{i j}^{t} & =w\left(\Pi_{i j, t}\right)=\bigoplus_{\mu=1}^{m} w\left(\Pi_{i j, t}^{\mu}\right), \quad t \geq n, \\
a_{i j}^{\mu, t} & =\bigoplus_{\nu=\mu}^{m} w\left(\Pi_{i j, t}^{\nu}\right), \quad t \geq n .
\end{aligned}
$$

Denote

$$
\mathcal{N}_{\mu}^{(t)}:=C_{\mu} S_{\mu}^{t} R_{\mu} .
$$

These $C S R$ products are defined from $A_{\mu}$ and $\mathcal{C}_{\mu}$ in the same way as $\mathcal{P}^{(t)}$, see Section 3, were defined from $A$ and $\mathcal{C}$, and it follows that the sequence $\mathcal{N}_{\mu}^{(t)}$ is periodic with period $\gamma_{\mu}$. Further denote by $\tau_{\mu}$ the greatest cyclicity of a component in $\mathcal{C}_{\mu}$ and by $n_{\mu}$ the number of nodes in $K_{\mu}$. The following is a version of Theorem 3.3 for $\mathcal{N}_{\mu}^{(t)}$.

Theorem 4.1 ( $\mu$-Heavy paths). Let $T_{\mu}$ be such that $\left\{S_{\mu}^{t}, t \geq T_{\mu}\right\}$ is periodic.

1. For $t \geq 0$,

$$
w\left(\Pi_{i j, t}^{\mu}\right) \leq \lambda_{\mu}^{t}\left(\mathcal{N}_{\mu}^{(t)}\right)_{i j} .
$$

2. For $t \geq T_{\mu}+2 \tau_{\mu}\left(n_{\mu}-1\right)$,

$$
w\left(\Pi_{i j, t}^{\mu}\right) \geq \lambda_{\mu}^{t}\left(\mathcal{N}_{\mu}^{(t)}\right)_{i j} .
$$

Proof. We can w.l.o.g. assume that $\lambda_{\mu}=1$, since both (4.6) and (4.7) are stable under scalar multiplication of $A$. After this, the claim follows from Theorem 3.3 .

In the theorem above, we can choose $T_{\mu}$ equal to each other and of the order $O\left(n^{2}\right)$ for all $\mu$. The main result of this section now immediately follows from Theorem 4.1 and (4.4), noting that $T_{\mu}+2 \tau_{\mu}\left(n_{\mu}-1\right) \leq 3 n^{2}$ for all $\mu$.

Theorem 4.2 (Nachtigall expansion). Let $A \in \mathbb{R}_{+}^{n \times n}$. Then for all $t \geq 3 n^{2}$,

$$
A_{\mu}^{t}=\bigoplus_{\nu=\mu}^{m} \lambda_{\nu}^{t} \mathcal{N}_{\nu}^{(t)}
$$

In particular,

$$
A^{t}=\bigoplus_{\nu=1}^{m} \lambda_{\nu}^{t} \mathcal{N}_{\nu}^{(t)}
$$



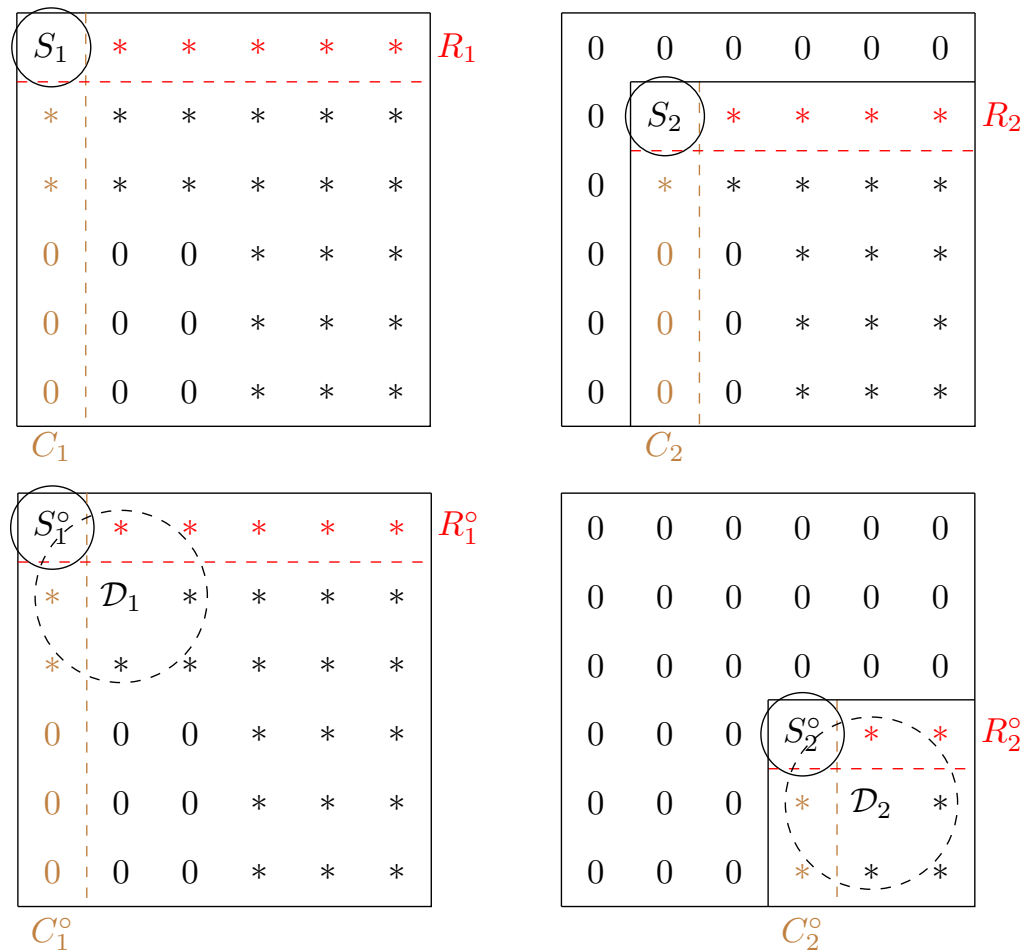

FiguRe 3. Formation of first two terms in a Nachtigall expansion (upper part) and ultimate expansion (lower part): a schematic example. The associated digraph consists of two components, denoted $\mathcal{D}_{1}$ and $\mathcal{D}_{2}$.

\section{Ultimate EXPANSION}

In this section we construct a different expansion of $A^{t}$, which we call the ultimate expansion, in order to describe the ultimate behavior of $A^{t}$. This expansion is related to the Nachtigall expansion of Section 4 with the selection rule $\mathcal{C}_{\mu}:=\mathcal{C}\left(A_{\mu}\right)$. The latter expansion will be called the canonical Nachtigall expansion.

The elements of the ultimate expansion will be labeled by $\circ$, since we need to distinguish them from those of the canonical Nachtigall expansion. For instance, we will write $A_{\mu}^{\circ}$ versus $A_{\mu}, \mathcal{C}_{\mu}^{\circ}$ versus $\mathcal{C}_{\mu}$ and $\lambda_{\mu}^{\circ}$ versus $\lambda_{\mu}$, etc.

Let $A=\left(a_{i j}\right) \in \mathbb{R}_{+}^{n \times n}$. Define $\lambda_{1}^{\circ}=\lambda(A)$, let $\mathcal{C}_{1}^{\circ}=\left(N_{1}^{\circ}, E_{1}^{\circ}\right)$ be the critical graph of $A$ and denote by $M_{1}^{\circ}$ the set of nodes in all components of $\mathcal{D}(A)$ that contain the components of $\mathcal{C}_{1}^{\circ}$. Set $A_{1}^{\circ}:=A$ and $K_{1}^{\circ}:=N$.

By induction for $\mu \geq 2$, define $K_{\mu}^{\circ}:=N \backslash \bigcup_{i=1}^{\mu-1} M_{i}^{\circ}\left(\right.$ instead of $K_{\mu}=N \backslash \bigcup_{i=1}^{\mu-1} N_{i}$ in the case of a Nachtigall expansion), and define $A_{\mu}^{\circ}=\left(a_{i j}^{\mu \circ}\right) \in \mathbb{R}_{+}^{n \times n}$ by

$$
a_{i j}^{\mu \circ}= \begin{cases}a_{i j}, & \text { if } i, j \in K_{\mu}^{\circ} \\ 0, & \text { otherwise }\end{cases}
$$


Define $\lambda_{\mu}^{\circ}:=\lambda\left(A_{\mu}^{\circ}\right)$. If $\lambda^{\circ}=0$, then stop; otherwise, let $\mathcal{C}_{\mu}^{\circ}=\left(N_{\mu}^{\circ}, E_{\mu}^{\circ}\right)$ be the critical graph of $A_{\mu}^{\circ}$, let $M_{\mu}^{\circ}$ be the set of all nodes in the components of $\mathcal{D}(A)$ which contain the components of $\mathcal{C}_{\mu}^{\circ}$, and proceed with the above definition for $\mu:=\mu+1$.

By the above procedure we define $K_{\mu}^{\circ}, A_{\mu}^{\circ}, \lambda_{\mu}^{\circ}, \mathcal{C}_{\mu}^{\circ}=\left(N_{\mu}^{\circ}, E_{\mu}^{\circ}\right)$ and $M_{\mu}^{\circ}$ for $\mu=1, \ldots, m^{\circ}$, where $m^{\circ} \leq n$ is the last number $\mu$ such that $\lambda_{\mu}^{\circ} \neq 0$. Note that $\left\{\lambda_{\mu}^{\circ}, \mu=1, \ldots, m^{\circ}\right\}$ is the set of m.c.g.m. of all nontrivial components of $\mathcal{D}(A)$, and each $\mathcal{C}_{\mu}^{\circ}$ consists of the critical digraphs of (possibly several) such components.

Let $\gamma_{\mu}^{\circ}$ be the cyclicity of $\mathcal{C}_{\mu}^{\circ}$, and let $B_{\mu}^{\circ}, C_{\mu}^{\circ}, S_{\mu}^{\circ}$ and $R_{\mu}^{\circ}$ be defined from $\left(A_{\mu}^{\circ} / \lambda_{\mu}^{\circ}\right)^{\gamma_{\mu}^{\circ}}$ in full analogy with $B_{\mu}, C_{\mu}, S_{\mu}$ and $R_{\mu}$ in Section 4 . The matrices $S_{\mu}^{\circ}$ can again be simultaneously scaled to $0-1$ form.

Essentially in the new construction we contract by the components of $\mathcal{D}(A)$ instead of the components of $\mathcal{C}\left(A_{\mu}\right)$ in the case of the canonical Nachtigall expansion. See Figure 3 for a visual comparison.

Denote by $\lambda(i)$ the m.c.g.m. of the component of $\mathcal{D}(A)$ to which $i$ belongs, and let $\lambda(i)=0$ if $\{i\}$ is a trivial component of $\mathcal{D}(A)$. For a path $P$ define $\lambda(P):=$ $\max _{i \in N_{P}} \lambda(i)$, where $N_{P}$ is the set of nodes visited by $P$. Recall that $\Pi_{i j, t}$ denotes the set of paths on $\mathcal{D}(A)$ which connect $i$ to $j$ and have length $t$. Denote by $\Pi_{i j, t}^{\mu \circ}$ the set of paths $P \in \Pi_{i j, t}$ such that $P$ contains a node in $N_{\mu}^{\circ}$ and $\lambda(P)=\lambda_{\mu}$. Such paths will be called $\mu$-hard. Note that they are $\mathcal{C}\left(A_{\mu}^{\circ}\right)$-heavy with respect to $A_{\mu}^{\circ}$.

Denote

$$
\mathcal{U}_{\mu}^{(t)}:=C_{\mu}^{\circ}\left(S_{\mu}^{\circ}\right)^{t} R_{\mu}^{\circ}
$$

These $C S R$ products are defined from $A_{\mu}^{\circ}$ and $\mathcal{C}_{\mu}^{\circ}$ in the same way as $\mathcal{P}^{(t)}$ were defined in Section 3 from $A$ and $\mathcal{C}$, and it follows that the sequence $\left\{\mathcal{U}_{\mu}^{(t)}, t \geq 0\right\}$ has the period $\gamma_{\mu}^{\circ}$. Denote by $\tau_{\mu}^{\circ}$ the greatest cyclicity of a component in $\mathcal{C}_{\mu}^{\circ}$ and by $n_{\mu}^{\circ}$ the number of nodes in $K_{\mu}^{\circ}$. The next result follows from Theorem 3.3 .

Theorem 5.1 ( $\mu$-Hard paths). Let $T_{\mu}^{\circ}$ be such that $\left\{\left(S_{\mu}^{\circ}\right)^{t}, t \geq T_{\mu}^{\circ}\right\}$ is periodic.

1. For $t \geq 0$,

$$
w\left(\Pi_{i j, t}^{\mu \circ}\right) \leq\left(\lambda_{\mu}^{\circ}\right)^{t}\left(\mathcal{U}_{\mu}^{t}\right)_{i j}
$$

2. For $t \geq T_{\mu}^{\circ}+2 \tau_{\mu}^{\circ}\left(n_{\mu}^{\circ}-1\right)$,

$$
w\left(\Pi_{i j, t}^{\mu \circ}\right) \geq\left(\lambda_{\mu}^{\circ}\right)^{t}\left(\mathcal{U}_{\mu}^{t}\right)_{i j} .
$$

Comparing the constructions above with those of the canonical Nachtigall expansion (see Section 4 assuming that $\mathcal{C}_{\mu}:=\mathcal{C}\left(A_{\mu}\right)$ ), we see that $\mathcal{C}_{1}$ is the same as $\mathcal{C}_{1}^{\circ}$ and $\lambda_{1}$ is the same as $\lambda_{1}^{\circ}$; however, other components and values may not be the same. We next describe the relation between them.

Proposition 5.2. Each $\nu=1, \ldots, m^{\circ}$ corresponds to a unique $\mu=1, \ldots, m$ such that $\lambda_{\nu}^{\circ}=\lambda_{\mu}$ and all components of $\mathcal{C}_{\nu}^{\circ}$ are also components of $\mathcal{C}_{\mu}$.

Proof. Consider the canonical Nachtigall expansion. Note that the $\lambda_{\mu}$ strictly decrease, as at each step of the definition we remove the whole critical digraph. Now pick arbitrary $\lambda_{\nu}^{\circ}$, which is the m.c.g.m. of some component of $\mathcal{D}(A)$. There is a reduction step when $\mathcal{C}_{\mu}$ for the first time intersects with a component of $\mathcal{D}(A)$ whose m.c.g.m. is $\lambda_{\nu}^{\circ}$. Then $\lambda_{\mu}=\lambda_{\nu}^{\circ}$, and $\mathcal{C}_{\mu}$ has to contain all components of $\mathcal{C}_{\nu}^{\circ}$, precisely as they are. This proves the claim. 
Further we renumber $\lambda_{\nu}^{\circ}$ so that $\nu=\mu$ if $\lambda_{\nu}^{\circ}=\lambda_{\mu}$, meaning that the numbering of $\lambda_{\nu}^{\circ}$ is adjusted to that of $\lambda_{\mu}$. This defines a subset $\Sigma$ of $\{1, \ldots, m\}$, such that $\lambda_{\mu}=\lambda_{\mu}^{\circ}$ if and only if $\mu \in \Sigma$.

Corollary 5.3. $\gamma_{\mu}$ is a multiple of $\gamma_{\mu}^{\circ}$ for each $\mu \in \Sigma$.

Proof. With the new numbering, all components of $\mathcal{C}_{\mu}^{\circ}$ are also components of $\mathcal{C}_{\mu}$ by Proposition 5.2 .

Unlike $\mu$-heavy paths, $\mu$-hard paths do not cover the whole path sets $\Pi_{i j, t}$ in general. However, evidently

$$
\left(A_{\mu}^{\circ}\right)_{i j}^{t}=w\left(\Pi_{i j, t}^{\mu \circ}\right) \quad \text { if } i \in N_{\mu}^{\circ} \text { or } j \in N_{\mu}^{\circ} .
$$

From this and Theorem 5.1 we deduce the following.

Proposition 5.4. Let $A \in \mathbb{R}_{+}^{n \times n}$. For all $t \geq 3 n^{2}$,

$$
\left(A_{\mu}^{\circ}\right)_{i j}^{t}=\lambda_{\mu}^{t} \mathcal{U}_{\mu}^{(t)} \quad \text { if } i \in N_{\mu}^{\circ} \text { or } j \in N_{\mu}^{\circ} .
$$

For the sequel we need to establish some relation between connectivity on $\mathcal{D}(A)$ and nonzero entries of $\mathcal{U}_{\mu}^{(t)}$.

We denote by $\gamma^{\circ}$ the l.c.m. of all cyclicities $\gamma_{\mu}^{\circ}$ of all components $\mathcal{C}_{\mu}^{\circ}$. Recall that we denote by $N_{P}$ the set of nodes visited by a path $P$ and by $\lambda(P)$ the greatest m.c.g.m. of a component visited by $P$.

Proposition 5.5. Let $i, j \in N, l \geq 0$ and $\mu \in \Sigma$. The following are equivalent.

1. $\left(\mathcal{U}_{\mu}^{(l)}\right)_{i j} \neq 0$.

2. For all $t \equiv l\left(\bmod \gamma^{\circ}\right)$ such that $t \geq 3 n^{2}$, there is a $\mu$-hard path of length $t$ connecting $i$ to $j$.

3. For some $t \equiv l\left(\bmod \gamma^{\circ}\right)$ there exists a $\mu$-hard path of length $t$ connecting $i$ to $j$.

4. For some $t \equiv l\left(\bmod \gamma^{\circ}\right)$ there exists a path $P$ of length $t$ such that $\lambda(P)=$ $\lambda_{\mu}$.

Proof. Implications $1 \Leftrightarrow 2$ and $3 \Leftrightarrow 1$ follow from Theorem 5.1 and the periodicity of $\mathcal{U}_{\mu}^{(t)}$. Implications $2 \Rightarrow 3 \Rightarrow 4$ are evident. It remains to prove $4 \Rightarrow 3$. Let $k$ be a node in $N_{P}$ which belongs to $M_{\mu}^{\circ}$, and let $l$ be a node in $N_{\mu}^{\circ}$ (that is, a critical node) in the same component of $\mathcal{D}(A)$ as $k$. There exists a cycle containing both $k$ and $l$. Adjoining $\gamma^{\circ}$ copies of this cycle to $P$ we obtain a $\mu$-hard path, whose length is congruent to $l\left(\bmod \gamma^{\circ}\right)$.

Now we establish the ultimate expansion of matrix powers, as an ultimate form of the canonical Nachtigall expansion. We will write $a(t) \stackrel{T}{=} b(t)$ if $a(t)=b(t)$ for all $t \geq t^{\prime}$, where $t^{\prime}$ is an unknown integer, and analogously for inequalities.

Theorem 5.6 (Ultimate expansion). Let $A \in \mathbb{R}_{+}^{n \times n}$. For all $\mu \in \Sigma$,

$$
\left(A_{\mu}^{\circ}\right)^{t} \stackrel{T}{=} \bigoplus_{\nu \in \Sigma: \nu \geq \mu} \lambda_{\nu}^{t} \mathcal{U}_{\nu}^{(t)}
$$

In particular,

$$
A^{t} \stackrel{T}{=} \bigoplus_{\nu \in \Sigma} \lambda_{\nu}^{t} \mathcal{U}_{\nu}^{(t)}
$$


Proof. It suffices to prove (5.8). First note that $\mathcal{U}_{\mu}^{(t)} \leq \mathcal{N}_{\mu}^{(t)}$ for all $\mu \in \Sigma$, since any $\mu$-hard path is a $\mu$-heavy path. As $A^{t} \stackrel{T}{=} \bigoplus_{\mu} \lambda_{\mu}^{t} \mathcal{N}_{\mu}^{(t)}$ by the canonical Nachtigall expansion, it suffices to prove that

$$
\bigoplus_{\mu=1}^{m} \lambda_{\mu}^{t} \mathcal{N}_{\mu}^{(t)} \stackrel{T}{\leq} \bigoplus_{\mu \in \Sigma} \lambda_{\mu}^{t} \mathcal{U}_{\mu}^{(t)}
$$

For all $\mu, i$ and $j$ such that $\left(\mathcal{N}_{\mu}^{(t)}\right)_{i j} \neq 0$, we will show that either $\mu \in \Sigma$ and $\left(\mathcal{U}_{\mu}^{(t)}\right)_{i j}=\left(\mathcal{N}_{\mu}^{(t)}\right)_{i j}$, or there exists $\nu \in \Sigma$ such that $\lambda_{\nu}>\lambda_{\mu}$ and $\left(\mathcal{U}_{\nu}^{(t)}\right)_{i j} \neq 0$.

Assume that either $\mu \notin \Sigma$, or $\mu \in \Sigma$ but $\left(\mathcal{N}_{\mu}^{(t)}\right)_{i j}>\left(\mathcal{U}_{\mu}^{(t)}\right)_{i j}$. Theorem 4.1 implies that for all $l$ such that $l \geq 3 n^{2}$ and $l \equiv t\left(\bmod \gamma_{\mu}\right)$ there exist paths $P \in \Pi_{i j, l}^{\mu}$ such that $w(P)=\lambda_{\mu}^{l}\left(\mathcal{N}_{\mu}^{(t)}\right)_{i j}$. We are going to show that these paths are not $\mu$-hard. If $\mu \notin \Sigma$, then this is immediate. If $\mu \in \Sigma$, then by Corollary 5.3, $\gamma_{\mu}$ is a multiple of $\gamma_{\mu}^{\circ}$, and hence $l \equiv t\left(\bmod \gamma_{\mu}^{\circ}\right)$. If $P$ is $\mu$-hard, then $w(P) \leq \lambda_{\mu}^{l}\left(\mathcal{U}_{\mu}^{(t)}\right)_{i j}$ by Theorem 5.1. which implies $\left(\mathcal{U}_{\mu}^{(t)}\right)_{i j} \geq\left(\mathcal{N}_{\mu}^{(t)}\right)_{i j}$, contradicting our assumptions. Hence $P$ is not $\mu$ hard, meaning that for any such path there exists $\nu \in \Sigma$ such that $\lambda_{\nu}=\lambda(P)>\lambda_{\mu}$. Applying Proposition [5.5] we obtain that $\left(\mathcal{U}_{\nu}^{(t)}\right)_{i j} \neq 0$ with $\lambda_{\nu}>\lambda_{\mu}$. The claim is proved.

If $A$ is irreducible, then the ultimate expansion has only one term, which corresponds to its critical graph $\mathcal{C}(A)$. In general, it has several terms (up to $n$ ) corresponding to the critical graphs of the components of $\mathcal{D}(A)$ (or possibly clusters of critical graphs of the components with the same m.c.g.m.). Thus, Theorem 5.6. can be regarded as a generalization of the Cyclicity Theorem (see [17, Theorem 3.9 or [1, Theorem 3.108), which it implies as a special irreducible case.

\section{Computational complexity}

Given $A \in \mathbb{R}_{+}^{n \times n}$, we investigate the computational complexity of the following problems.

(P1) For given $t$, reconstruct all terms $\mathcal{N}_{\mu}^{(t)}$ of a Nachtigall expansion with a prescribed selection rule for $\mathcal{C}_{\mu}$.

(P2) For given $t: 0 \leq t<\gamma$, reconstruct all terms $\mathcal{U}_{\mu}^{(t)}$ of the ultimate expansion.

In (P1) we assume that selecting the subdigraph $\mathcal{C}_{\mu}$ of $\mathcal{C}\left(A_{\mu}\right)$ does not take more than $O\left(n^{3}\right)$ operations. This holds in particular if $\mathcal{C}_{\mu}$ is an arbitrary cycle of $\mathcal{C}\left(A_{\mu}\right)$ as in [19, 21].

Problem (P1) is close to the problem considered by Molnárová [19, and Problem (P2) is an extension of a problem considered by Sergeev 25]. An $O\left(n^{4} \log n\right)$ solution of these problems is given below. It is based on visualization, square multiplication and permutation of cyclic classes. See Semančíková [22, 23] for closely related studies in max-min algebra.

Theorem 6.1. For any $A \in \mathbb{R}_{+}^{n \times n}$, problems (P1) and (P2) can be solved in $O\left(n^{4} \log n\right)$ operations.

Proof. (P1): First we need to compute $\lambda_{\mu}, A_{\mu}$ and $\mathcal{C}_{\mu}$ for all $\mu$. At each step the computation requires no more than $O\left(n^{3}\right)$ operations, based on Karp and FloydWarshall methods applied to each component of $\mathcal{D}\left(A_{\mu}\right)$. The total complexity is no more than $O\left(n^{4}\right)$. After this, we find all cyclic classes in each $\mathcal{C}_{\mu}$, which has total 
complexity $O\left(n^{2}\right)$, and hence the cyclicities $\gamma_{\sigma}$ of all components of the graphs $\mathcal{C}_{\mu}$. At this stage we can also find a scaling which leads to a total $S$-visualization of $A$ (and hence all $A_{\mu}$ ). This relies on the Floyd-Warshall method applied to each $S_{\mu}$ and takes no more than $O\left(n^{3}\right)$ operations in total.

By Theorem 4.2, the $A_{\mu}^{t}$ admit a Nachtigall expansion for all $\mu$ and all $t \geq 3 n^{2}$. The rows and columns of $A_{\mu}^{t}$ with indices in $N_{\mu}$ are determined at $t \geq 3 n^{2}$ only by $\mathcal{N}_{\mu}^{t}=C_{\mu} S_{\mu}^{t} R_{\mu}$, since by construction these rows and columns are zero in all terms $\mathcal{N}_{\nu}^{(t)}$ for $\nu>\mu$. This means in particular that these rows and columns become periodic after $3 n^{2}$ time. By repeated squaring $A_{\mu}, A_{\mu}^{2}, A_{\mu}^{4}, \ldots$, we reach a power $A_{\mu}^{r}$ with $r \geq 3 n^{2}$, which requires no more than $O\left(n^{3} \log n\right)$ operations. Now we can use Corollary 3.7 identifying $C_{\mu} S_{\mu}^{r}$ and $S_{\mu}^{r} R_{\mu}$ as submatrices extracted from columns, resp. rows, of $A_{\mu}^{t}$ with indices in $N_{\mu}$. By Theorem 3.6 we can obtain $S_{\mu}^{t} R_{\mu}$ from $S_{\mu}^{r} R_{\mu}$ and $C_{\mu}$ from $C_{\mu} S_{\mu}^{r}$ by the permutation on cyclic classes determined by the remainders $r\left(\bmod \gamma_{\sigma}\right)$ and $(t-r)\left(\bmod \gamma_{\sigma}\right)$, for each cyclicity $\gamma_{\sigma}$ of a component of $\mathcal{C}_{\mu}$. This takes $O\left(n^{2}\right)$ overrides. Finally we compute $\mathcal{N}_{\mu}^{t}=C_{\mu} S_{\mu}^{t} R_{\mu}$ $\left(O\left(n^{3}\right)\right.$ operations). We conclude that the total complexity for all $\mu$ does not exceed $O\left(n^{4} \log n\right)$ operations.

(P2): It is clear that the computation of all prerequisites for the ultimate expansion is done as in the first paragraph of the proof of (P1), and takes no more than $O\left(n^{4}\right)$ operations. After that we use Proposition 5.4 which means that the critical rows and columns in each $\left(A_{\mu}^{\circ}\right)^{r}$ for $r \geq 3 n^{2}$ are determined only by $\mathcal{U}_{\mu}^{(r)}$. Hence the factors of each $\mathcal{U}_{\mu}^{(t)}$ can be computed by matrix squaring of $A_{\mu}^{\circ}$, followed by a permutation on cyclic classes and matrix multiplication, which overall takes no more than $O\left(n^{3} \log n\right)$ operations. We conclude that the total complexity for all $\mu$ does not exceed $O\left(n^{4} \log n\right)$ operations.

\section{ORBIT PERIODIC MATRICES}

Being motivated by the results of Butkovič et al. [6] on robust matrices, we are going to derive necessary and sufficient conditions for orbit periodicity and show that they can be verified in polynomial time.

A matrix $A \in \mathbb{R}_{+}^{n \times n}$ is called orbit periodic if for each $y \in \mathbb{R}_{+}^{n}$ there exists $\lambda(y) \in \mathbb{R}_{+}^{n}$ such that $A^{t+\gamma^{\circ}} y=(\lambda(y))^{\gamma^{\circ}} A^{t} y$ for all sufficiently large $t$, where (as above) $\gamma^{\circ}$ is the joint cyclicity (l.c.m.) of the critical graphs of all components of $\mathcal{D}(A)$.

A sequence $\left\{A^{t} y, t \geq 0\right\}$ with the above property will be called ultimately linear periodic and $\lambda(y)$ will be called its ultimate growth rate. The same wording will be used for the sequences $\left\{a_{i j}^{t}, t \geq 0\right\}$. We say that a subsequence $\left\{a_{i j}^{l+\gamma^{\circ} s}, s \geq 0\right\}$ has ultimate growth rate $\lambda$ if there exists $\alpha_{i j} \neq 0$ such that $a_{i j}^{l+\gamma^{\circ} s}=\alpha_{i j} \lambda^{l+\gamma^{\circ} s}$ for all $s$ starting from a sufficiently large number.

It may seem more general if in the above definition of linear periodicity we replace $\gamma^{\circ}$ by $\gamma(y)$. But using the ultimate expansion (5.8) we conclude that $\left\{A^{t} y, t \geq 0\right\}$ is ultimately linear periodic if and only if there exists $\mu \in \Sigma$ such that $A^{t} y \stackrel{T}{=} \lambda_{\mu}^{t} \mathcal{U}_{\mu}^{(t)} y$. As $\mathcal{U}_{\mu}^{\left(t+\gamma^{\circ}\right)}=\mathcal{U}_{\mu}^{(t)}$ for all $\mu$ and $t$, we conclude that the exact period of $\left\{A^{t} y, t \geq 0\right\}$ has to divide $\gamma^{\circ}$.

The ultimate expansion leads to the following properties of the sequences $\left\{a_{i j}^{t}\right.$, $t \geq 0\}$, already known in max algebra [9, 16, 20. As above, $\lambda(P)$ is the largest 
m.c.g.m. of the components of $\mathcal{D}(A)$ visited by $P$, and $\Pi_{i j, t}$ denotes the set of paths of length $t$ connecting $i$ to $j$.

Lemma 7.1. For each $l: 0 \leq l<\gamma^{\circ}$, the subsequence $\left\{a_{i j}^{l+\gamma^{\circ} s}, s \geq 0\right\}$ is ultimately zero or has an ultimate growth rate $\lambda_{i j}(l)$. In the latter case, for each such $l$ and each sufficiently large $t \equiv l\left(\bmod \gamma^{\circ}\right)$ there exists $P \in \Pi_{i j, t}$ such that $\lambda(P)=\lambda_{i j}(l)$.

Proof. The ultimate expansion (5.8) implies that for each $l$ there exists $\mu \in \Sigma$ such that $a_{i j}^{l+\gamma^{\circ} s}=\lambda_{\mu}^{l+\gamma^{\circ} s}\left(\mathcal{U}_{\mu}^{(l)}\right)_{i j}$ at sufficiently large $s$, so the subsequence has growth rate $\lambda_{i j}(l):=\lambda_{\mu}$. The second part of the statement follows from Proposition 5.5 .

Lemma 7.2. If $P \in \Pi_{i j, l}$, then $\left\{a_{i j}^{l+\gamma^{\circ} s}, s \geq 0\right\}$ has ultimate growth rate at least $\lambda(P)$.

Proof. Using Proposition [5.5, part 4, we obtain that $\left(\mathcal{U}_{\mu}^{(l)}\right)_{i j} \neq 0$ for $\mu$ such that $\lambda_{\mu}=\lambda(P)$, and then $a_{i j}^{l+\gamma^{\circ} s} \geq(\lambda(P))^{l+\gamma^{\circ} s}\left(\mathcal{U}_{\mu}^{(l)}\right)_{i j}$ at sufficiently large $s$.

Denote by $L^{\circ}$ the set of nodes in the nontrivial components of $\mathcal{D}(A)$. The next statement follows from the Cyclicity Theorem [1, 17. For the sake of completeness we deduce it from the ultimate expansion.

Lemma 7.3. If $i, j \in L^{\circ}$ belong to the same component of $\mathcal{D}(A)$, then $\left\{a_{i j}^{t}, t \geq 0\right\}$ is ultimately linear periodic and its growth rate is the m.c.g.m. of that component. Further, for each $i \in L^{\circ}$ and all $t$ there exist $k$ and $l$ in the same component of $\mathcal{D}(A)$ such that $a_{i k}^{t} \neq 0$ and $a_{l i}^{t} \neq 0$.

Proof. All paths which connect $i$ to $j$ are $\mu$-hard, with $\lambda_{\mu}=\lambda(i)=\lambda(j)$. Using Theorem 5.1 we obtain that only $\left(\mathcal{U}_{\mu}^{(t)}\right)_{i j}$ is nonzero and hence the ultimate expansion reads $a_{i j}^{t} \stackrel{T}{=} \lambda_{\mu}^{t}\left(\mathcal{U}_{\mu}^{(t)}\right)_{i j}$. For the second part, note that $i$ belongs to a cycle with nonzero weight.

If $i$ is connected to $j$ by a path, we denote this by $i \rightarrow j$. Observe that if $i \rightarrow j$, then also $k \rightarrow l$ for each $k$ in the same component of $\mathcal{D}(A)$ with $i$ and for each $l$ in the same component of $\mathcal{D}(A)$ with $j$. We also denote $i \leftrightarrow j$ if both $i \rightarrow j$ and $j \rightarrow i$ (i.e., if $i$ and $j$ are in the same component of $\mathcal{D}(A)$ ). In the next theorem we describe, in terms of such relations, when the sequences of columns $\left\{A^{t} e_{i}, t \geq 0\right\}$ are ultimately linear periodic.

Proposition 7.4. Let $A \in \mathbb{R}_{+}^{n \times n}$ and $j \in L^{\circ}$. The sequence $\left\{A^{t} e_{j}, t \geq 0\right\}$ is ultimately linear periodic if and only if for all $i \in L^{\circ}, i \rightarrow j$ implies $\lambda(i) \leq \lambda(j)$.

Proof. The "only if" part: Lemma 7.3 implies that for each $t$ there exists $k$ such that $a_{k j}^{t} \neq 0$, and the sequence $\left\{a_{k j}^{t+s \gamma^{\circ}}, s \geq 0\right\}$ has ultimate growth rate $\lambda(j)$. If the condition does not hold, there exists a path $P$ leading from $i$ to $j$ such that $\lambda(P)=\lambda(i)>\lambda(j)$, and by Lemma 7.2 there is a subsequence of $\left\{a_{i j}^{l}, l \geq 0\right\}$ with ultimate growth rate at least $\lambda(i)$.

The "if" part: If the sequence $\left\{A^{t} e_{j}, t \geq 0\right\}$ is not ultimately linear periodic, then some of its entries by Lemma 7.3 have ultimate growth rate $\lambda(j)$ and there is a subsequence of $\left\{a_{k j}^{t}, t \geq 0\right\}$, for some $k \in N$, which has a different ultimate growth rate. Lemma 7.1 implies that this growth rate has to be greater than $\lambda(j)$ and must be the m.c.g.m. of a component which has access to $j$. 
We denote $i \rightrightarrows j$ and say that $i$ strongly accesses $j$ if $i$ can be connected to $j$ by a path of any length starting from a certain number $T_{i j}$. For example, $i$ strongly accesses $j$ if $i \rightarrow j$ and the cyclicities of the components of $\mathcal{D}(A)$ containing $i$ and $j$ are coprime. Observe that if $i \rightrightarrows j$, then also $k \rightrightarrows l$ for each $k \leftrightarrow i$ and $l \leftrightarrow j$. Now we show that strong access relations are essential for the ultimate linear periodicity of all sequences $\left\{A^{t}\left(e_{i} \oplus e_{j}\right), t \geq 0\right\}$.

Proposition 7.5. Let $A \in \mathbb{R}_{+}^{n \times n}$ and $i, j \in L^{\circ}$. Suppose that $\left\{A^{t} e_{i}, t \geq 0\right\}$ and $\left\{A^{t} e_{j}, t \geq 0\right\}$ are ultimately linear periodic. Then $\left\{A^{t}\left(e_{k} \oplus e_{l}\right), t \geq 0\right\}$ is also ultimately linear periodic for all $k \leftrightarrow i$ and $l \leftrightarrow j$ if and only if $i \rightrightarrows j$, or $j \rightrightarrows i$, or $\lambda(i)=\lambda(j)$.

Proof. If $\lambda(i)=\lambda(j)$, then both $\left\{A^{t} e_{k}, t \geq 0\right\}$ and $\left\{A^{t} e_{l}, t \geq 0\right\}$ for all $k \leftrightarrow i$ and $l \leftrightarrow j$ have this growth rate and $\left\{A^{t}\left(e_{k} \oplus e_{l}\right), t \geq 0\right\}$ is ultimately linear periodic with this growth rate. So it remains to consider the case $\lambda(i)<\lambda(j)$. In this case Proposition 7.4 implies $j \not \rightarrow i$, and therefore we have to show that $\left\{A^{t}\left(e_{k} \oplus e_{l}\right), t \geq 0\right\}$ are ultimately linear periodic for all $k \leftrightarrow i$ and $l \leftrightarrow j$ if and only if $i \rightrightarrows j$.

The "if" part: For each $t: 0 \leq t<\gamma^{\circ}$, if there exists $m \in N$ and $s_{1} \geq 0$ such that $a_{m k}^{t+s_{1} \gamma^{\circ}} \neq 0$, then there exists a path $P \in \Pi_{m k, t+s_{1} \gamma^{\circ}}$. As $k \rightrightarrows l$, this path can be joined with a path from $k$ to $l$ of length $s_{2} \gamma^{\circ}$, and we get that $a_{m l}^{t+\left(s_{1}+s_{2}\right) \gamma^{\circ}} \neq 0$. Using Lemma 7.2 we obtain $a_{m l}^{t+s \gamma^{\circ}} \neq 0$ for all sufficiently large $s$ and it dominates over $a_{m k}^{t+s \gamma^{\circ}}$ since it has larger growth rate.

The "only if" part: The ultimate linear periodicity of $\left\{A^{t}\left(e_{k} \oplus e_{j}\right), t \geq 0\right\}$, for any $k \leftrightarrow i$, implies that $\operatorname{supp}\left(A^{t} e_{k}\right) \subseteq \operatorname{supp}\left(A^{t} e_{j}\right)$ for all large enough $t$. By Lemma 7.3 there is $k \leftrightarrow i$ such that $\left(A^{t}\right)_{i k} \neq 0$; hence also $\left(A^{t}\right)_{i j} \neq 0$. As we reasoned for any $t$, it follows that $i \rightrightarrows j$.

Theorem 7.6 (Orbit periodicity). $A \in \mathbb{R}_{+}^{n \times n}$ is orbit periodic if and only if the following conditions hold for all $i, j \in L^{\circ}$ :

1. $i \rightarrow j$ implies $\lambda(i) \leq \lambda(j)$,

2. if neither $j \rightrightarrows i$ nor $i \rightrightarrows j$, then $\lambda(i)=\lambda(j)$,

or equivalently if $\left\{A^{t}\left(e_{i} \oplus e_{j}\right), t \geq 0\right\}$ are ultimately linear periodic for all $i, j \in L^{\circ}$.

Proof. We need only prove that 1 and 2 are sufficient for orbit periodicity. The rest relies on Propositions 7.4 and 7.5 .

Let $\mathcal{D}(A, y)$ be the subgraph induced by the set of nodes that have access to $\operatorname{supp}(y):=\left\{i: y_{i} \neq 0\right\}$. Nontrivial components $\mathcal{D}_{\sigma}$ of $\mathcal{D}(A, y)$ are ordered by relation $\mathcal{D}_{\sigma_{1}} \preceq \mathcal{D}_{\sigma_{2}}$ if $i \rightrightarrows j$ for some (and hence all) $i \in \mathcal{D}_{\sigma_{1}}$ and $j \in \mathcal{D}_{\sigma_{2}}$. Consider the maximal components with respect to this relation. By conditions 1 and 2 they must have the same m.c.g.m. and it must be the greatest one. We denote this m.c.g.m. by $\lambda$ and show that it is the ultimate growth rate of $A^{t} y$.

By Lemma 7.1 for each $l: 0 \leq l<\gamma$, the subsequence $\left\{a_{i j}^{l+s \gamma^{\circ}}, s \geq 0\right\}$ has a certain ultimate growth rate if it is not ultimately zero. We have to show that $\lambda$ is the maximal growth rate of $\left\{a_{i k}^{l+s \gamma^{\circ}}, s \geq 0\right\}$ over $k \in \operatorname{supp}(y)$, for every fixed $i \in N$ and $l: 0 \leq l<\gamma^{\circ}$. Then it follows that $A^{t+\gamma^{\circ}} y \stackrel{T}{=} \lambda^{\gamma^{\circ}} A^{t} y$.

To avoid trivialities we assume that there exists $k \in \operatorname{supp}(y)$ and such that $\left\{a_{i k}^{l+s \gamma^{\circ}}, s \geq 0\right\}$ is not ultimately zero. Then for some $k \in \operatorname{supp}(y)$ there exists a path $P \in \Pi_{i k, t}$ where $t \equiv l\left(\bmod \gamma^{\circ}\right)$ which visits a nontrivial component $\mathcal{D}_{\sigma_{1}}$ 
of $\mathcal{D}(A, y)$. If the m.c.g.m. of $\mathcal{D}_{\sigma_{1}}$ is $\lambda$, then by Lemma 7.2 the growth rate of $\left\{a_{i k}^{l+s \gamma^{\circ}}, s \geq 0\right\}$ is not less than $\lambda$; hence it must be $\lambda$ and we are done. If the m.c.g.m. of $\mathcal{D}_{\sigma_{1}}$ is less than $\lambda$, then $\mathcal{D}_{\sigma_{1}}$ strongly accesses some component $\mathcal{D}_{\sigma_{2}}$ with m.c.g.m. $\lambda$, and $\mathcal{D}_{\sigma_{2}}$ accesses a node $k^{\prime} \in \operatorname{supp}(y)$. Due to the strong access we can adjust the length of the path from $\mathcal{D}_{\sigma_{1}}$ to $\mathcal{D}_{\sigma_{2}}$ if necessary, and we obtain a path $P^{\prime} \in \Pi_{i k^{\prime}, t^{\prime}}$ where $t^{\prime} \equiv l\left(\bmod \gamma^{\circ}\right)$. By Lemma 7.2 we obtain that the growth rate of $\left\{a_{i k^{\prime}}^{l+s \gamma}, s \geq 0\right\}$ is not less than $\lambda$; hence it must be $\lambda$.

To assess the computational feasibility of condition 2 in Theorem 7.6 we need the following observation which uses the ultimate expansion; see Theorem 5.6 and Section 5 .

Theorem 7.7. Let $A \in \mathbb{R}_{+}^{n \times n}$ be such that the sequences $\left\{A^{t} e_{i}, t \geq 0\right\}$ are ultimately linear periodic for all $i \in L^{\circ}$. Then $A$ is orbit periodic if and only if the following holds for all $\mu, \nu \in \Sigma$ :

$$
\lambda_{\mu}<\lambda_{\nu} \Rightarrow \bigcup_{i \in N_{\mu}^{\circ}} \operatorname{supp}\left(\mathcal{U}_{\mu}^{(1)} e_{i}\right) \subseteq \bigcap_{j \in N_{\nu}^{\circ}} \operatorname{supp}\left(\mathcal{U}_{\nu}^{(1)} e_{j}\right)
$$

Proof. If $\left\{A^{t} e_{i}, t \geq 0\right\}$ is ultimately linear periodic and $i \in L^{\circ}$, then $A^{t} e_{i} \stackrel{T}{=}$ $\lambda_{\mu}^{t} \mathcal{U}_{\mu}^{(t)} e_{i}$ for $\lambda_{\mu}=\lambda(i)$, since $\lambda_{\mu}$ must be the growth rate of $A^{t} e_{i}$ and all other terms of the ultimate expansion have different growth rates. Then also $A^{t}\left(e_{i} \oplus e_{j}\right) \stackrel{T}{=}$ $\lambda_{\mu}^{t} \mathcal{U}_{\mu}^{(t)} e_{i} \oplus \lambda_{\nu}^{t} \mathcal{U}_{\nu}^{(t)} e_{j}$, where $\lambda_{\mu}=\lambda(i)$ and $\lambda_{\nu}=\lambda(j)$ (equivalently, $i \in M_{\mu}^{\circ}$ and $\left.j \in M_{\nu}^{\circ}\right)$. If $\lambda_{\mu}<\lambda_{\nu}$, then $\left\{A^{t}\left(e_{i} \oplus e_{j}\right), t \geq 0\right\}$ is ultimately linear periodic if and only if $A^{t}\left(e_{i} \oplus e_{j}\right) \stackrel{T}{=} \lambda_{\nu}^{t} \mathcal{U}_{\nu}^{(t)} e_{j}$. This happens if and only if

$$
\lambda_{\mu}<\lambda_{\nu} \Rightarrow \operatorname{supp}\left(\mathcal{U}_{\mu}^{(t)} e_{i}\right) \subseteq \operatorname{supp}\left(\mathcal{U}_{\nu}^{(t)} e_{j}\right) \forall i \in M_{\mu}^{\circ}, j \in M_{\nu}^{\circ}
$$

holds for all $\mu, \nu \in \Sigma$.

Corollary 3.8 implies that any column of $\mathcal{U}_{\mu}^{(t)}$, resp. $\mathcal{U}_{\nu}^{(t)}$, is a max-linear combination of columns with indices in $N_{\mu}^{\circ}$, resp. $N_{\nu}^{\circ}$, so that the support of that column is the union of supports of certain columns with indices in $N_{\mu}^{\circ}$, resp. $N_{\nu}^{\circ}$. Hence we need to check the support inclusions only for $i \in N_{\mu}^{\circ}$ and $j \in N_{\nu}^{\circ}$. Further, Corollary 3.7 implies that the columns of $\mathcal{U}_{\mu}^{(t)}$ (or resp. $\mathcal{U}_{\nu}^{(t)}$ ) with indices in $N_{\mu}^{\circ}$ (or resp. $N_{\nu}^{\circ}$ ) just permute as $t$ changes, so the inclusions need be verified only for $t=1$. This shows that (17.2) is equivalent to

$$
\lambda_{\mu}<\lambda_{\nu} \Rightarrow \operatorname{supp}\left(\mathcal{U}_{\mu}^{(1)} e_{i}\right) \subseteq \operatorname{supp}\left(\mathcal{U}_{\nu}^{(1)} e_{j}\right) \forall i \in N_{\mu}^{\circ}, j \in N_{\nu}^{\circ},
$$

which is equivalent to (7.1). The claim is proved.

Now we give a polynomial bound on the computational complexity of verifying the orbit periodicity of a reducible matrix.

Theorem 7.8. Let $A \in \mathbb{R}_{+}^{n \times n}$. Suppose that all components of $\mathcal{D}(A)$ and access relations between them are known, and $\lambda_{\mu}$ and $\mathcal{U}_{\mu}^{(1)}$ are computed for all $\mu \in \Sigma$. Then the orbit periodicity of $A$ can be verified in no more than $O\left(n^{3}\right)$ operations.

Proof. For all pairs $\mu, \nu$, we must verify condition 1 of Theorem 7.6 and condition (17.1). The first of these conditions is verified for the pairs of components of $\mathcal{D}(A)$ and it takes no more than $O\left(n^{2}\right)$ if all access relations between them are known. To verify condition (7.1), we need to compute all unions $\bigcup_{i \in N_{\mu}^{\circ}} \operatorname{supp}\left(\mathcal{U}_{\mu}^{(1)} e_{i}\right)$ and 
intersections $\bigcap_{i \in N_{\mu}^{\circ}} \operatorname{supp}\left(\mathcal{U}_{\mu}^{(1)} e_{i}\right)$, which requires $O\left(n^{2}\right)$ operations, and then make $O\left(n^{2}\right)$ comparisons of Boolean vectors, which requires no more than $O\left(n^{3}\right)$ operations.

We combine the results of Theorems 6.1 and 7.8 .

Corollary 7.9. Given $A \in \mathbb{R}_{+}^{n \times n}$, it takes no more than $O\left(n^{4} \log n\right)$ operations to verify whether it is orbit periodic or not.

\section{EXAMPLES}

All examples in this section will be in the max-plus setting $\mathbb{R}_{\max ,+}:=(\mathbb{R} \cup$ $\{-\infty\}, \oplus=\max , \otimes=+)$.

Example 1. We construct the canonical Nachtigall expansion of $A^{t}$ for

$$
A=\left(\begin{array}{cccc}
-1 & 0 & -7 & -6 \\
0 & -1 & -5 & -4 \\
-7 & -5 & -1 & -3 \\
-6 & -4 & -3 & -2
\end{array}\right) .
$$

We start with $A_{1}=A$. The maximal cycle mean is $\lambda_{1}=0$, and the component $\mathcal{C}_{1}$ has two nodes 1,2 and two edges $(1,2)$ and $(2,1)$. The cyclicity is $\gamma_{1}:=2$.

We proceed by setting the entries in the first two rows and columns to $-\infty$, thus obtaining

$$
A_{2}=\left(\begin{array}{cccc}
-\infty & -\infty & -\infty & -\infty \\
-\infty & -\infty & -\infty & -\infty \\
-\infty & -\infty & -1 & -3 \\
-\infty & -\infty & -3 & -2
\end{array}\right) .
$$

The maximum cycle mean is $\lambda_{2}=-1$, and the component $\mathcal{C}_{2}$ has one node 3 and one edge $(3,3)$. The cyclicity is $\gamma_{2}=1$.

Now we set everything to $-\infty$ except for the entry $(4,4)$ :

$$
A_{3}=\left(\begin{array}{cccc}
-\infty & -\infty & -\infty & -\infty \\
-\infty & -\infty & -\infty & -\infty \\
-\infty & -\infty & -\infty & -\infty \\
-\infty & -\infty & -\infty & -2
\end{array}\right) .
$$

The maximum cycle mean is $\lambda_{3}=-2$, and the component $\mathcal{C}_{3}$ has one node 4 and one edge $(4,4)$. The cyclicity is $\gamma_{3}=1$.

We obtain matrices $S_{1}, S_{2}$ and $S_{3}$, which correspond, respectively, to $\mathcal{C}_{1}, \mathcal{C}_{2}$ and $\mathcal{C}_{3}$ :

$$
\begin{aligned}
S_{1} & =\left(\begin{array}{cccc}
-\infty & 0 & -\infty & -\infty \\
0 & -\infty & -\infty & -\infty \\
-\infty & -\infty & -\infty & -\infty \\
-\infty & -\infty & -\infty & -\infty
\end{array}\right), S_{2}=\left(\begin{array}{cccc}
-\infty & -\infty & -\infty & -\infty \\
-\infty & -\infty & -\infty & -\infty \\
-\infty & -\infty & 0 & -\infty \\
-\infty & -\infty & -\infty & -\infty
\end{array}\right), \\
S_{3} & =\left(\begin{array}{cccc}
-\infty & -\infty & -\infty & -\infty \\
-\infty & -\infty & -\infty & -\infty \\
-\infty & -\infty & -\infty & -\infty \\
-\infty & -\infty & -\infty & 0
\end{array}\right) .
\end{aligned}
$$

These are Boolean matrices in the max-plus setting, with entries $\infty, 0$ instead of 0,1 . 
Further we need to compute Kleene stars $\left(A^{\gamma_{1}}\right)^{*},\left(\left(A_{2}-\lambda_{2}\right)^{\gamma_{2}}\right)^{*}=\left(1+A_{2}\right)^{*}$ and $\left(\left(A_{3}-\lambda_{3}\right)^{\gamma_{3}}\right)^{*}=\left(2+A_{3}\right)^{*}$, and construct matrices $C_{1}, R_{1}, C_{2}, R_{2}, C_{3}$ and $R_{3}$. The critical parts of the Kleene stars are shown below, the rest of the elements being denoted by $\cdot$ as we do not need them:

$$
\begin{aligned}
& \left(A^{2}\right)^{*}=\left(\begin{array}{cccc}
0 & -1 & -5 & -4 \\
-1 & 0 & -6 & -5 \\
-5 & -6 & \cdot & \cdot \\
-4 & -5 & \cdot & \cdot
\end{array}\right), \\
& \left(1 \otimes A_{2}\right)^{*}=\left(\begin{array}{cccc}
\cdot & \cdot & -\infty & \cdot \\
\cdot & \cdot & -\infty & \cdot \\
-\infty & -\infty & 0 & -2 \\
\cdot & \cdot & -2 & \cdot
\end{array}\right), \quad\left(2 \otimes A_{3}\right)^{*}=\left(\begin{array}{cccc}
\cdot & \cdot & \cdot & -\infty \\
\cdot & \cdot & \cdot & -\infty \\
\cdot & \cdot & \cdot & -\infty \\
-\infty & -\infty & -\infty & 0
\end{array}\right) .
\end{aligned}
$$

Further we compute the Nachtigall matrices:

$$
\begin{aligned}
& \mathcal{N}_{1}^{(0)}=C_{1} \otimes R_{1}=\left(\begin{array}{cccc}
0 & -1 & -5 & -4 \\
-1 & 0 & -6 & -5 \\
-5 & -6 & -10 & -9 \\
-4 & -5 & -9 & -8
\end{array}\right) \\
& \mathcal{N}_{1}^{(1)}=C_{1} \otimes S_{1} \otimes R_{1}=\left(\begin{array}{cccc}
-1 & 0 & -6 & -5 \\
0 & -1 & -5 & -4 \\
-6 & -5 & -11 & -10 \\
-5 & -4 & -10 & -9
\end{array}\right), \\
& \mathcal{N}_{2}^{(0)}=C_{2} \otimes R_{2}=\left(\begin{array}{cccc}
-\infty & -\infty & -\infty & -\infty \\
-\infty & -\infty & -\infty & -\infty \\
-\infty & -\infty & 0 & -2 \\
-\infty & -\infty & -2 & -4
\end{array}\right) \\
& \mathcal{N}_{3}^{(0)}=C_{3} \otimes R_{3}=\left(\begin{array}{cccc}
-\infty & -\infty & -\infty & -\infty \\
-\infty & -\infty & -\infty & -\infty \\
-\infty & -\infty & -\infty & -\infty \\
-\infty & -\infty & -\infty & 0
\end{array}\right)
\end{aligned}
$$

The Nachtigall expansion starts to work already at $t=2$. Indeed,

$$
\begin{aligned}
A^{2} & =\left(\begin{array}{cccc}
0 & -1 & -5 & -4 \\
-1 & 0 & -6 & -5 \\
-5 & -6 & -2 & -4 \\
-4 & -5 & -4 & -4
\end{array}\right)=\mathcal{N}_{1}^{(0)} \oplus(-2) \otimes \mathcal{N}_{2}^{(0)} \oplus(-4) \otimes \mathcal{N}_{3}^{(0)} \\
A^{3} & =\left(\begin{array}{cccc}
-1 & 0 & -6 & -5 \\
0 & -1 & -5 & -4 \\
-6 & -5 & -3 & -5 \\
-5 & -4 & -5 & -6
\end{array}\right)=\mathcal{N}_{1}^{(1)} \oplus(-3) \otimes \mathcal{N}_{2}^{(0)} \oplus(-6) \otimes \mathcal{N}_{3}^{(0)}
\end{aligned}
$$

Starting from $t=4$ the third term can be forgotten:

$$
A^{4}=\left(\begin{array}{cccc}
0 & -1 & -5 & -4 \\
-1 & 0 & -6 & -5 \\
-5 & -6 & -4 & -6 \\
-4 & -5 & -6 & -8
\end{array}\right)=\mathcal{N}_{1}^{(0)} \oplus(-4) \otimes \mathcal{N}_{2}^{(0)} .
$$


The ultimate periodic behavior starts after $T(A)=10$ :

$$
A^{10}=\left(\begin{array}{cccc}
0 & -1 & -5 & -4 \\
-1 & 0 & -6 & -5 \\
-5 & -6 & -10 & -9 \\
-4 & -5 & -9 & -8
\end{array}\right)=\mathcal{N}_{1}^{(0)}=\mathcal{U}_{1}^{(0)} .
$$

Example 2. The following example will illustrate the ultimate expansion:

$$
A=\left(\begin{array}{ccccccc}
-2 & 0 & -3 & -7 & -\infty & -\infty & -\infty \\
0 & -2 & -5 & -7 & -\infty & -\infty & -\infty \\
-9 & -7 & -9 & -8 & -\infty & -\infty & -\infty \\
-9 & -6 & -4 & -4 & -\infty & -\infty & -\infty \\
-8 & -5 & -5 & -4 & -1 & -7 & -5 \\
-7 & -8 & -5 & -6 & -3 & -6 & -8 \\
-6 & -4 & -9 & -3 & -5 & -5 & -5
\end{array}\right) .
$$

We compute the elements of the ultimate expansion.

First, $A_{1}^{\circ}=A, \lambda_{1}=0$, and the component $\mathcal{C}_{1}^{\circ}=\mathcal{C}(A)$ consists of two nodes 1,2 and two edges $(1,2)$ and $(2,1)$.

On the next step we set all entries in the first four rows and columns of $A$ to $-\infty$, thus obtaining matrix $A_{2}^{\circ}$. Its essential submatrix with finite entries is extracted from the remaining rows and columns 5 to 7 :

$$
A_{2 \text { ess }}^{\circ}=\left(\begin{array}{lll}
-1 & -7 & -5 \\
-3 & -6 & -8 \\
-5 & -5 & -5
\end{array}\right), \quad 5 \text { to } 7 \times 5 \text { to } 7 .
$$

We compute $\lambda_{2}=-1$, and the component $\mathcal{C}_{2}=\mathcal{C}\left(A_{2}\right)$ consists of the loop $(5,5)$.

The components $\mathcal{C}_{1}$ and $\mathcal{C}_{2}$ determine Boolean (i.e., $\left.0,-\infty\right)$ matrices $S_{1}$ and $S_{2}$. We compute $\left(A^{2}\right)^{*}$ and $\left(1 \otimes A_{2}^{\circ}\right)^{*}$ :

$$
\begin{aligned}
& \left(A^{2}\right)^{*}=\left(\begin{array}{ccccccc}
0 & -2 & -5 & -7 & -\infty & -\infty & -\infty \\
-2 & 0 & -3 & -7 & -\infty & -\infty & -\infty \\
-7 & -9 & 0 & -12 & -\infty & -\infty & -\infty \\
-6 & -8 & -8 & 0 & -\infty & -\infty & -\infty \\
-5 & -6 & -6 & -5 & 0 & -8 & -6 \\
-8 & -7 & -8 & -7 & -4 & 0 & -8 \\
-4 & -6 & -7 & -7 & -6 & -10 & 0
\end{array}\right), \\
& \left(1 \otimes A_{2 \text { ess }}^{\circ}\right)^{*}=\left(\begin{array}{ccc}
0 & -6 & -4 \\
-2 & 0 & -6 \\
-4 & -4 & 0
\end{array}\right), \quad 5 \text { to } 7 \times 5 \text { to } 7 .
\end{aligned}
$$

Next we build matrices $C_{1}^{\circ}, R_{1}^{\circ}$ and $C_{2}^{\circ}, R_{2}^{\circ}$ whose essential parts are shown below:

$$
\begin{aligned}
C_{1 \text { ess }}^{\circ} & =\left(\begin{array}{ccccccc}
0 & -2 & -7 & -6 & -5 & -8 & -4 \\
-2 & 0 & -9 & -8 & -6 & -7 & -6
\end{array}\right)^{T}, 1 \text { to } 7 \times 1 \text { to } 2, \\
R_{1 \text { ess }}^{\circ} & =\left(\begin{array}{cccc}
0 & -2 & -5 & -7 \\
-2 & 0 & -3 & -7
\end{array}\right), 1 \text { to } 2 \times 1 \text { to } 4,
\end{aligned}
$$




$$
\begin{aligned}
& C_{2 \text { ess }}^{\circ}=\left(\begin{array}{lll}
0 & -2 & -4
\end{array}\right)^{T}, \quad 5 \text { to } 7 \times 5, \\
& R_{2 \text { ess }}^{\circ}=\left(\begin{array}{lll}
0 & -6 & -4
\end{array}\right), \quad 5 \times 5 \text { to } 7 .
\end{aligned}
$$

Using (8.14) and (8.15) we compute the ultimate terms $\mathcal{U}_{1}^{(0)}=C_{1}^{\circ} \otimes R_{1}^{\circ}, \mathcal{U}_{1}^{(1)}=$ $C_{1}^{\circ} \otimes S_{1}^{\circ} \otimes R_{1}^{\circ}$ and $\mathcal{U}_{2}^{(0)}=C_{2}^{\circ} \otimes R_{2}^{\circ}$. The ultimate expansion starts to work at $t=9$, meaning

$$
\begin{aligned}
A^{9} & =\mathcal{U}_{1}^{(1)} \oplus(-9) \otimes \mathcal{U}_{2}^{(0)}, A^{10}=\mathcal{U}_{1}^{(0)} \oplus(-10) \otimes \mathcal{U}_{2}^{(0)}, \\
A^{11} & =\mathcal{U}_{1}^{(1)} \oplus(-11) \otimes \mathcal{U}_{2}^{(0)}, A^{12}=\mathcal{U}_{1}^{(0)} \oplus(-12) \otimes \mathcal{U}_{2}^{(0)}, \ldots
\end{aligned}
$$

In this case, the canonical Nachtigall expansion already starts to work at $t=3$, and after $t=4$ only the first two terms are essential. In this expansion, matrix $A_{2}$ results from setting only the first two (instead of four) columns and rows to $-\infty$. The second Nachtigall term $\mathcal{N}_{2}$ is equal to $C_{2} \otimes R_{2}$, where $C_{2}=C_{2}^{\circ}$ and $R_{2} \neq R_{2}^{\circ}$ has the essential part

$$
R_{2 \text { ess }}=\left(\begin{array}{lllll}
-4 & -3 & 0 & -6 & -4
\end{array}\right), \quad 5 \times 3 \text { to } 7 .
$$

Example 3. We illustrate the total periodicity. Let

$$
\begin{aligned}
A & =\left(\begin{array}{cccccc}
-\infty & 1 & -\infty & -\infty & -\infty & -\infty \\
-\infty & -\infty & 1 & -\infty & -\infty & -\infty \\
-\infty & -\infty & -\infty & 1 & -\infty & -\infty \\
1 & -\infty & -\infty & -\infty & -\infty & -\infty \\
-\infty & -2 & -\infty & -\infty & -\infty & 0 \\
-\infty & -2 & -\infty & -\infty & 0 & -\infty
\end{array}\right), \\
B & =\left(\begin{array}{cccccc}
-\infty & 1 & -\infty & -\infty & -\infty & -\infty \\
-\infty & -\infty & 1 & -\infty & -\infty & -\infty \\
-\infty & -\infty & -\infty & 1 & -\infty & -\infty \\
1 & -\infty & -\infty & -\infty & -\infty & -\infty \\
-\infty & -2 & -\infty & -\infty & -\infty & 0 \\
-\infty & -\infty & -2 & -\infty & 0 & -\infty
\end{array}\right) .
\end{aligned}
$$

Note that $A$ and $B$ are almost the same, except for the entries $(6,2)$ and $(6,3)$. In both cases the ultimate expansion coincides with the canonical Nachtigall expansion, and we have two critical components $\mathcal{C}_{1}=\left(N_{1}, E_{1}\right)$ with $N_{1}=\{1,2,3,4\}$, $E_{1}=\{(1,2),(2,3),(3,4),(4,1)\}$ and $\mathcal{C}_{2}=\left(N_{2}, E_{2}\right)$ with $N_{2}=\{5,6\}$ and $E_{2}=$ $\{(5,6),(6,5)\}$. The eigenvalues are $\lambda_{1}=1$ and $\lambda_{2}=0$, and the cyclicities are $\gamma_{1}=4$ and $\gamma_{2}=2$; their l.c.m. is $\gamma=4$. Note that $M_{1}^{\circ}=N_{1}$ and $M_{2}^{\circ}=N_{2}$.

In both cases the component with $\lambda_{1}$ does not have access to the component with $\lambda_{2}$, which is smaller; hence condition 1 of Theorem 7.6 is true, meaning that all columns of $A^{t}$ and $B^{t}$ are ultimately periodic. Condition 2 of Theorem 7.6 holds 
for $A$, but it does not hold for $B$. In particular, there are only paths of odd length connecting node 6 to node 3 . Hence $A$ is orbit periodic and $B$ is not.

Also consider condition (7.1). We need the terms of the ultimate expansion for $t\left(\bmod \gamma^{\circ}\right)=1$. In each case there are two terms, which we denote by $\mathcal{U}_{1}^{A}$ and $\mathcal{U}_{2}^{A}$, resp. $\mathcal{U}_{1}^{B}$ and $\mathcal{U}_{2}^{B}$, for the case of $A$, resp. $B$. We have

$$
\begin{aligned}
& \mathcal{U}_{1}^{A}=\left(\begin{array}{cccccc}
-\infty & 0 & -\infty & -\infty & -\infty & -\infty \\
-\infty & -\infty & 0 & -\infty & -\infty & -\infty \\
-\infty & -\infty & -\infty & 0 & -\infty & -\infty \\
0 & -\infty & -\infty & -\infty & -\infty & -\infty \\
-4 & -3 & -6 & -5 & -\infty & -\infty \\
-4 & -3 & -6 & -5 & -\infty & -\infty
\end{array}\right) \\
& \mathcal{U}_{2}^{B}=\left(\begin{array}{cccccc}
-\infty & 0 & -\infty & -\infty & -\infty & -\infty \\
-\infty & -\infty & 0 & -\infty & -\infty & -\infty \\
-\infty & -\infty & -\infty & 0 & -\infty & -\infty \\
0 & -\infty & -\infty & -\infty & -\infty & -\infty \\
-\infty & -3 & -\infty & -5 & -\infty & -\infty \\
-4 & -\infty & -3 & -\infty & -\infty & -\infty
\end{array}\right), \\
& \mathcal{U}_{2 \text { ess }}^{A}=\mathcal{U}_{2}^{B} \text { ess }=\left(\begin{array}{cc}
-\infty & 0 \\
0 & -\infty
\end{array}\right), 5 \text { to } 6 \times 5 \text { to } 6 .
\end{aligned}
$$

Observe that $\operatorname{supp}\left(\mathcal{U}_{2}^{A} e_{i}\right) \subseteq \operatorname{supp}\left(\mathcal{U}_{1}^{A} e_{j}\right)$ for all $i \in M_{2}^{\circ}=\{5,6\}$ and $j \in M_{1}^{\circ}=$ $\{1,2,3,4\}$, but this condition does not hold for $B$.

To see the difference between $A^{t} x$ and $B^{t} x$, take $x=\left[\begin{array}{ll}0-\infty-\infty-\infty-\infty & -\infty\end{array}\right]^{T}$. The sequence $\left\{A^{t} x\right\}$ is ultimately periodic starting from $t=4$ with period 4 and growth rate 1 . In particular the last component of $\left\{A^{t} x\right\}$ yields the following number sequence for $t \geq 4$ :

$$
\left(A^{t} x\right)_{6}=\{111155559999 \ldots\}, t \geq 4 .
$$

The sequence $\left\{B^{t} x\right\}$ is not ultimately periodic. In particular, the last component of $\left\{B^{t} x\right\}$ yields the following number sequence for $t \geq 2$ :

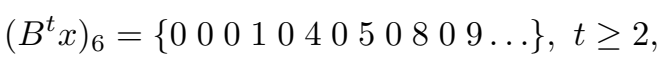

which can be expressed as

$$
\left(B^{t} x\right)_{6}= \begin{cases}0, & \text { if } t \text { is even and } t \geq 2 \\ t-3, & \text { if } t=4 k+3 \text { and } k \geq 0 \\ t-5, & \text { if } t=4 k+5 \text { and } k \geq 0\end{cases}
$$

\section{ACKNOWLEDGEMENT}

We thank Peter Butkovič for his encouraging support, numerous discussions and careful reading of the paper. We acknowledge the work of the anonymous reviewer, who helped us to eliminate some mistakes and typos. We are also grateful to Abdulhadi Aminu, Trivikram Dokka and Glenn Merlet for their comments, help, and advice. 


\section{REFERENCES}

[1] F.L. Baccelli, G. Cohen, G.-J. Olsder, and J.-P. Quadrat, Synchronization and linearity: an algebra for discrete event systems, Wiley, 1992. MR1204266 (94b:93001)

[2] Y. Balcer and A.F. Veinott, Computing a graph's period quadratically by node condensation, Discrete Mathematics 4 (1973), 295-303. MR0327566 (48:5908)

[3] R.A. Brualdi and H.J. Ryser, Combinatorial matrix theory, Cambridge Univ. Press, 1991. MR1130611 (93a:05087)

[4] P. Butkovič, Max-algebra: the linear algebra of combinatorics?, Linear Algebra Appl. 367 (2003), 313-335. MR1976928 (2004d:15006)

[5] L Max-linear systems: theory and algorithms, Springer-Verlag, London, 2010. MR2681232 (2011e:15049)

[6] P. Butkovič, R. A. Cuninghame-Green, and S. Gaubert, Reducible spectral theory with applications to the robustness of matrices in max-algebra, SIAM J. on Matrix Anal. and Appl. 31 (2009), no. 3, 1412-1431. MR2587784 (2011b:15061)

[7] J. Cochet-Terrasson, S. Gaubert, and J. Gunawardena, A constructive fixed-point theorem for min-max functions, Dynamics and Stability of Systems 14 (1999), no. 4, 407-433. MR:1746112 (2000m:47077)

[8] R. A. Cuninghame-Green, Minimax algebra, Lecture Notes in Economics and Mathematical Systems, vol. 166, Springer, Berlin, 1979. MR.580321 (82a:90043)

[9] B. de Schutter, On the ultimate behavior of the sequence of consecutive powers of a matrix in the max-plus algebra, Linear Algebra Appl. 307 (2000), 103-117. MR1741919 (2000j:15045)

[10] L. Elsner and P. van den Driessche, On the power method in max algebra, Linear Algebra Appl. 302-303 (1999), 17-32. MR.1733521 (2000k:15021)

[11] _ Modifying the power method in max algebra, Linear Algebra Appl. 332-334 (2001), 3-13. MR1839423 (2002c:15037)

[12] G.M. Engel and H. Schneider, Cyclic and diagonal products on a matrix, Linear Algebra Appl. 7 (1973), 301-335. MR0323804 (48:2160)

[13] M. Fiedler and V. Pták, Diagonally dominant matrices, Czechoslovak Math. J. 17 (1967), no. 92, 420-433. MR0215869 (35:6704)

[14] G. Frobenius, Über Matrizen aus nicht negativen Elementen, Sitzber. Preuss. Akad. Wiss. (1912), 456-477.

[15] M. Gavalec, Linear matrix period in max-plus algebra, Linear Algebra Appl. 307 (2000), 167-182. MR1741924 (2000m:15020)

[16] _ Periodicity in extremal algebra, Gaudeamus, Hradec Králové, 2004.

[17] B. Heidergott, G.-J. Olsder, and J. van der Woude, Max-plus at work, Princeton Univ. Press, 2005.

[18] K.H. Kim, Boolean matrix theory and applications, Marcel Dekker, New York, 1982. MR.655414 (84a:15001)

[19] M. Molnárová, Computational complexity of Nachtigall's representation, Optimization 52 (2003), 93-104. MR1962942 (2004a:05097)

[20] _ Generalized matrix period in max-plus algebra, Linear Algebra Appl. 404 (2005), 345-366. MR2149669 (2006f:15018)

[21] K. Nachtigall, Powers of matrices over an extremal algebra with applications to periodic graphs, Mathematical Methods of Operations Research 46 (1997), 87-102. MR.1464921 (99e:05076)

[22] B. Semančíková, Orbits in max-min algebra, Linear Algebra Appl. 414 (2006), 38-63. MR2209233 (2006j:15057)

[23] - Orbits and critical components of matrices in max-min algebra, Linear Algebra Appl. 426 (2007), 415-447. MR2350666 (2009a:15063)

[24] B. Semančíková, Private communication, 2009.

[25] S. Sergeev, Max algebraic powers of irreducible matrices in the periodic regime: An application of cyclic classes, Linear Algebra Appl. 431 (2009), 1325-1339. MR2547914 (2010k:15056)

[26] S. Sergeev, H. Schneider, and P. Butkovič, On visualization scaling, subeigenvectors and Kleene stars in max algebra, Linear Algebra Appl., 431 (2009), no. 12, 2395-2406. MR2563030 (2010j:15028) 
[27] S̆. Schwartz, On a sharp estimation in the theory of binary relations on a finite set, Czechoslovak Math. J. 20 (1970), 703-714. MR0282862 (44:96)

[28] H. Wielandt, Unzerlegbare nicht negative Matrizen, Math. Z. 52 (1950), 642-645. MR0035265 $(11: 710 \mathrm{~g})$

School of Mathematics, University of Birmingham, Watson Building, Edgbaston B15 2TT, United KINGDOM

E-mail address: sergiej@gmail.com

Department of Mathematics, University of Wisconsin-Madison, Madison, Wisconsin 53706

E-mail address: hans@math.wisc.edu 